TITLE:

\title{
Non-collinear interaction of guided elastic waves in an isotropic plate
}

$\operatorname{AUTHOR}(S)$ :

Ishii, Yosuke; Biwa, Shiro; Adachi, Tadaharu

CITATION:

Ishii, Yosuke ... [et al]. Non-collinear interaction of guided elastic waves in an isotropic plate. Journal of Sound and Vibration 2018, 419: 390-404

ISSUE DATE:

2018-04-14

URL:

http://hdl.handle.net/2433/234665

RIGHT:

(c) 2018. This manuscript version is made available under the CC-BY-NC-ND 4.0 license

http://creativecommons.org/licenses/by-nc-nd/4.0/; The full-text file will be made open to the public on 14 April 2020 in accordance with publisher's 'Terms and Conditions for Self-Archiving'.; この論文は出版社版でありません。引用の際に は出版社版をご確認ご利用ください。; This is not the published version. Please cite only the published version. 
This manuscript has been published in:

Journal of Sound and Vibration, Vol. 419 (2018), pp. 390-404.

DOI: $10.1016 /$ j.jsv.2018.01.031

Non-collinear interaction of guided elastic waves in an isotropic plate

\author{
Yosuke Ishii ${ }^{\mathrm{a},}$, Shiro Biwa ${ }^{\mathrm{b}}$, and Tadaharu Adachi ${ }^{\mathrm{a}}$ \\ a Department of Mechanical Engineering, Toyohashi University of Technology, 1-1 \\ Hibarigaoka, Tempaku-cho, Toyohashi 441-8580, Japan \\ ${ }^{\mathrm{b}}$ Department of Aeronautics and Astronautics, Graduate School of Engineering, Kyoto \\ University, Katsura, Nishikyo-ku, Kyoto 615-8540, Japan
}

\begin{abstract}
The nonlinear wave propagation in a homogeneous and isotropic elastic plate is analyzed theoretically to investigate the non-collinear interaction of plate wave modes. In the presence of two primary plate waves (Rayleigh-Lamb or shear horizontal modes) propagating in arbitrary directions, an explicit expression for the modal amplitude of nonlinearly generated wave fields with the sum or difference frequency of the primary modes is derived by using the perturbation analysis. The modal amplitude is shown to grow in proportion with the propagation distance when the resonance condition is satisfied, i.e., when the wavevector of secondary wave coincides with the sum or difference of those of primary modes. Furthermore, the non-collinear interaction of two symmetric or two antisymmetric modes is shown to produce the secondary wave fields consisting only of the symmetric modes, while a pair of symmetric and antisymmetric primary modes is shown to produce only the antisymmetric modes. The influence of the intersection angle, the primary frequencies, and the mode combinations on the modal amplitude of secondary wave is examined for a low-frequency range where the lowest-order symmetric and antisymmetric Rayleigh-Lamb waves and the lowest-order symmetric shear horizontal wave are the only propagating modes.
\end{abstract}

Keywords: Non-collinear interaction; Rayleigh-Lamb wave; Shear horizontal wave; Perturbation analysis

* Corresponding author: Yosuke Ishii, E-mail address: ishii@me.tut.ac.jp 


\section{Introduction}

When two elastic waves intersect at certain angles, a third wave with a frequency and wavevector equal to the sum or difference of those of the intersecting waves is generated due to the material nonlinearity. This phenomenon is called non-collinear interaction and has attracted much attention as a promising tool for nondestructive testing, since it is advantageous compared to the conventional nonlinear acoustic techniques such as the use of higher-order harmonic generation [1-3]: in the conventional techniques, it is usually difficult to isolate signals due to the material nonlinearity to be detected since they are measured along with the incident wave which contains signals caused by the nonlinearities of measuring systems such as transducers and amplifiers. In contrast, the third wave generated by the non-collinear interaction propagates in different directions from the incident waves. Furthermore, it can have different frequency components from those of higher harmonics of incident waves. The signals due to the material nonlinearity can be hence more easily isolated from the system nonlinearities by appropriate choice of driving frequencies.

The non-collinear interaction has been studied for bulk waves in isotropic elastic media theoretically [4-8] as well as numerically $[9,10]$, leading to the derivation of the so-called resonance condition for the occurrence of third waves, i.e., the ratio of driving frequencies, the angle of intersection, and the combination of the incident and third wave modes. The third waves generated by the non-collinear interaction were experimentally observed by Rollins et al. [11], and the influence of applied stress on the generation behavior of third wave was investigated by Hirao et al. [12]. More recently, the amplitude of third waves has been shown to vary sensitively with the concentration of micro cracks in polymethyl methacrylate [9], fatigue damage of aluminum alloy [13], 
physical ageing of thermoplastics [14,15], and oxidative ageing state of concrete [16]. The non-collinear mixing method was also applied to a contacting interface between two solids for a sensitive evaluation of imperfect interfaces $[9,17,18]$. These foregoing works [4-18] dealt with the non-collinear interaction of non-dispersive bulk waves, i.e., pure longitudinal and pure shear waves.

For plate-like structures, which are widely used in aerospace, automotive, marine, and civil engineering, nonlinear acoustic phenomena have been extensively studied such as the higher-harmonic generation [19-34] and the collinear interaction [21,35,36] of guided waves, while the corresponding issue for the non-collinear interaction has rarely been addressed: Furgason and Newhouse [37] experimentally demonstrated that the non-collinear mixing of Lamb waves in a lead zirconate titanate plate could generate a third wave for certain combinations of Lamb modes. Since the behavior of non-collinear interaction in plate-like structures can be more complicated than that in bulk media due to their multimode as well as dispersive nature, in-depth theoretical investigation of this issue is important academically as well as practically to apply the non-collinear mixing method to the nondestructive evaluation of plate-like structures.

In this paper, the nonlinear wave propagation in a homogeneous and isotropic elastic plate is analyzed theoretically to elucidate the non-collinear interaction of guided waves. For the collinear mixing of two plate wave modes, a perturbation analysis for the third wave generation was presented in the two-dimensional framework by de Lima and Hamilton [21]. Extending this procedure for arbitrary propagation directions of primary waves, the generation behavior of third waves due to the non-collinear interaction is investigated. The formulation is laid down in Section 2, and an expression for the modal amplitude of the nonlinearly generated third wave is described in Section 3. The 
influence of the frequency, the intersection angle, and the primary wave modes on the modal amplitude is presented in Section 4 for the interaction of lowest-order plate wave modes.

It is noted that throughout this paper, the summation convention is used unless otherwise stated.

\section{Linearization of governing equations for a nonlinear isotropic plate}

The present study analyzes the three-dimensional nonlinear wave propagation in a plate of thickness $2 h$ with stress-free surfaces. When the Cartesian coordinate system $X_{1}-X_{2}-X_{3}$ is set in the reference configuration as shown in Fig. 1, the governing equations in the absence of body forces are given by

$$
\rho_{0} \ddot{U}_{j}\left(X_{1}, X_{2}, X_{3}, t\right)=P_{j k, k}\left(X_{1}, X_{2}, X_{3}, t\right), \quad-h<X_{3}<h, \quad j=1,2,3,
$$

where $\rho_{0}$ is the mass density of the plate, $U_{j}$ are the particle displacement components, and $t$ is the time. The overdot denotes the time derivative, and the subscript ", $k$ " denotes the spatial derivative with respect to $X_{k}$. Furthermore, $P_{j k}$ in Eq. (1) are the components of the first Piola-Kirchhoff stress tensor given by

$$
P_{j k}\left(X_{1}, X_{2}, X_{3}, t\right)=\left(\delta_{j l}+U_{j, l}\right) \frac{\partial W}{\partial E_{l k}}, \quad j, k=1,2,3
$$

where $\delta_{j l}$ is Kronecker's delta, $E_{l k}\left(X_{1}, X_{2}, X_{3}, t\right)=\left(U_{l, k}+U_{k, l}+U_{s, l} U_{s, k}\right) / 2(l, k=1,2,3)$ are the components of the Green-Lagrange strain tensor, and $W$ is the strain energy density of the plate. Assuming that the plate possesses isotropic elasticity with the quadratic nonlinearity, $W$ is expressed as 


$$
W=\frac{\lambda}{2} E_{l l} E_{r r}+\mu E_{l r} E_{r l}+\frac{\mathcal{A}}{3} E_{l r} E_{r s} E_{s l}+\mathcal{B} E_{l l} E_{r s} E_{s r}+\frac{\mathcal{C}}{3} E_{l l} E_{r r} E_{s S}
$$

where $\lambda$ and $\mu$ are Lamé's elastic constants, and $\mathcal{A}, \mathcal{B}$, and $\mathcal{C}$ are the third-order elastic constants $[38,39]$. The stress-free boundary conditions on the top $\left(X_{3}=h\right)$ and bottom $\left(X_{3}=-h\right)$ surfaces of the plate are given by

$$
P_{j 3}\left(X_{1}, X_{2}, \pm h, t\right)=0, \quad j=1,2,3 \text {. }
$$

Assuming that the nonlinearity is weak and that the solution of Eq. (1) is given as

$$
U_{j}=U_{j}^{\mathrm{L}}+U_{j}^{\mathrm{NL}}, \quad U_{k}^{\mathrm{L}} U_{k}^{\mathrm{L}} \gg U_{k}^{\mathrm{NL}} U_{k}^{\mathrm{NL}}, \quad j=1,2,3,
$$

where the superscripts "L" and "NL" denote the primary and secondary solutions, respectively, the governing equations for $U_{j}^{\mathrm{L}}$ and $U_{j}^{\mathrm{NL}}$ are given by performing the perturbation analysis for Eqs. (1)-(4) [21] as

For $U_{j}^{\mathrm{L}} ; \quad \rho_{0} \ddot{U}_{j}^{\mathrm{L}}=P_{j k, k}^{\mathrm{L}, \mathrm{L}}$,

$$
P_{j 3}^{\mathrm{L}, \mathrm{L}}\left(X_{1}, X_{2}, \pm h, t\right)=0, \quad j=1,2,3,
$$

For $U_{j}^{\mathrm{NL}} ; \quad \rho_{0} \ddot{U}_{j}^{\mathrm{NL}}=P_{j k, k}^{\mathrm{L}, \mathrm{NL}}+F_{j}^{\mathrm{NL}, \mathrm{L}}$,

$$
P_{j 3}^{\mathrm{L}, \mathrm{NL}}\left(X_{1}, X_{2}, \pm h, t\right)=-P_{j 3}^{\mathrm{NL}, \mathrm{L}}\left(X_{1}, X_{2}, \pm h, t\right), \quad j=1,2,3
$$

where the double sign in Eq. (7b) applies in the same order. In the above expressions, $P_{j k}^{\mathrm{L}, w}(w=\mathrm{L}, \mathrm{NL})$ and $P_{j k}^{\mathrm{NL}, \mathrm{L}}$ denote the linear and the quadratic terms of the stress tensor in terms of the displacement gradient, respectively, which are given as 


$$
\begin{aligned}
P_{j k}^{\mathrm{L}, w}= & \lambda U_{l, l}^{w} \delta_{j k}+\mu\left(U_{j, k}^{w}+U_{k, j}^{w}\right), \\
P_{j k}^{\mathrm{NL}, \mathrm{L}} & =\left(\frac{\lambda+\mathcal{B}}{2} U_{l, r}^{\mathrm{L}} U_{l, r}^{\mathrm{L}}+\frac{\mathcal{B}}{2} U_{l, r}^{\mathrm{L}} U_{r, l}^{\mathrm{L}}+\mathcal{C} U_{l, l}^{\mathrm{L}} U_{r, r}^{\mathrm{L}}\right) \delta_{j k}+\mathcal{B} U_{l, l}^{\mathrm{L}} U_{k, j}^{\mathrm{L}} \\
& +\frac{\mathcal{A}}{4} U_{l, j}^{\mathrm{L}} U_{k, l}^{\mathrm{L}}+(\lambda+\mathcal{B}) U_{l, l}^{\mathrm{L}} U_{j, k}^{\mathrm{L}}+\left(\mu+\frac{\mathcal{A}}{4}\right)\left(U_{j, l}^{\mathrm{L}} U_{k, l}^{\mathrm{L}}+U_{l, j}^{\mathrm{L}} U_{l, k}^{\mathrm{L}}+U_{j, l}^{\mathrm{L}} U_{l, k}^{\mathrm{L}}\right) .
\end{aligned}
$$

Furthermore, $F_{j}^{\mathrm{NL}, \mathrm{L}}$ in Eq. (7a) is given as

$$
\begin{aligned}
F_{j}^{\mathrm{NL}, \mathrm{L}} & =P_{j k, k}^{\mathrm{NL}, \mathrm{L}} \\
& =\left(\mu+\frac{\mathcal{A}}{4}\right)\left(U_{l, r r}^{\mathrm{L}} U_{l, j}^{\mathrm{L}}+U_{l, r r}^{\mathrm{L}} U_{j, l}^{\mathrm{L}}+2 U_{j, l r}^{\mathrm{L}} U_{l, r}^{\mathrm{L}}\right) \\
& +\left(\lambda+\mu+\frac{\mathcal{A}}{4}+\mathcal{B}\right)\left(U_{l, r j}^{\mathrm{L}} U_{l, r}^{\mathrm{L}}+U_{l, l r}^{\mathrm{L}} U_{j, r}^{\mathrm{L}}\right)+(\lambda+\mathcal{B}) U_{j, l l}^{\mathrm{L}} U_{r, r}^{\mathrm{L}} \\
& +\left(\frac{\mathcal{A}}{4}+\mathcal{B}\right)\left(U_{l, l r}^{\mathrm{L}} U_{r, j}^{\mathrm{L}}+U_{l, r j}^{\mathrm{L}} U_{r, l}^{\mathrm{L}}\right)+(\mathcal{B}+2 \mathcal{C}) U_{l, l j}^{\mathrm{L}} U_{r, r}^{\mathrm{L}} .
\end{aligned}
$$

The perturbation solution of Eqs. (1)-(4), $U_{j}$ in Eq. (5), can be sought by first solving the linear problem of Eq. (6a) in the absence of body forces with the stress-free boundary conditions of Eq. (6b) for the primary solution $U_{j}^{\mathrm{L}}$, and then solving Eqs. (7a) and (7b) for the secondary solution $U_{j}^{\mathrm{NL}}$, which are also linear but have a body force term as well as tractions on the plate surfaces consisting of the primary solution.

Note here that the present analysis focuses on the generation behavior of secondary wave fields which occur as the first-order perturbation of Eq. (5), while the tertiary and higher-order ones [36] can be analyzed by accounting for the next higher-order corrections in Eq. (5). 


\section{Non-collinear interaction of two plate modes}

\subsection{Primary solution}

Since the problem of Eq. (6) governs the wave propagation in a linear elastic isotropic plate, the primary solution $U_{j}^{\mathrm{L}}$ can be given as the superposition of four types of plate wave modes, i.e., symmetric and antisymmetric Rayleigh-Lamb modes, and symmetric and antisymmetric shear horizontal modes. In what follows, the generation behavior of wave fields due to the non-collinear interaction of two plate modes propagating in arbitrary directions is analyzed. Namely, the primary solution $U_{j}^{\mathrm{L}}$ is assumed to be written as

$$
\begin{gathered}
U_{j}^{\mathrm{L}}\left(X_{1}, X_{2}, X_{3}, t\right)=\frac{1}{2} \sum_{\alpha=1}^{2} A^{(\alpha)} R_{j l}^{-\theta^{(\alpha)}} \widehat{U}_{l}^{(\alpha)}\left(X_{3}\right) \exp \left\{\mathrm{i}\left[\kappa^{(\alpha)}\left(X_{1} \cos \theta^{(\alpha)}+X_{2} \sin \theta^{(\alpha)}\right)-\omega^{(\alpha)} t\right]\right\}+\text { c. c. } \\
j=1,2,3
\end{gathered}
$$

where the superscript $\alpha$ denotes the two primary wave modes, $i$ the imaginary unit, and c.c. the complex conjugate. In the above expression, $\kappa^{(\alpha)}$ and $\omega^{(\alpha)}$ are the wavenumber and the angular frequency, respectively, which satisfy any one of the following dispersion equations $[40,41]$ :

$$
\text { Rayleigh-Lamb modes: } \frac{\tan (q h)}{\tan (p h)}=-\left[\frac{4 \kappa^{2} p q}{\left(q^{2}-\kappa^{2}\right)^{2}}\right]^{ \pm 1}\left\{\begin{array}{l}
+1: \text { symmetric, } \\
-1: \text { antisymmetric, }
\end{array}\right.
$$

Shear horizontal modes: $q h=\frac{n \pi}{2}\left\{\begin{array}{l}n=0,2,4, \ldots: \text { symmetric, } \\ n=1,3,5, \ldots: \text { antisymmetric, }\end{array}\right.$

where 


$$
p=\sqrt{\left(\frac{\omega}{c_{\mathrm{L}}}\right)^{2}-\kappa^{2}}, \quad q=\sqrt{\left(\frac{\omega}{c_{\mathrm{T}}}\right)^{2}-\kappa^{2}}
$$

and $c_{\mathrm{L}}=\sqrt{(\lambda+2 \mu) / \rho_{0}}$ and $c_{\mathrm{T}}=\sqrt{\mu / \rho_{0}}$ are the longitudinal and shear wave velocity in the plate, respectively. The present study assumes that primary waves are both propagating modes with the positive frequency, i.e., $\operatorname{Im}\left[\kappa^{(\alpha)}\right]=0$ and $\omega^{(\alpha)}>0$. $\operatorname{In}$ Eq. (10), $\widehat{U}_{l}^{(\alpha)}$ represent the normalized displacement profile in the thickness direction when the corresponding plate wave mode propagates in the $X_{1}$ direction, which are given by [41]

Symmetric Rayleigh-Lamb modes:

$$
\begin{aligned}
& \widehat{U}_{1}\left(X_{3}\right)=\mathrm{i} \kappa\left[\frac{\cos \left(p X_{3}\right)}{\sin (p h)}+\frac{2 p q}{\kappa^{2}-q^{2}} \frac{\cos \left(q X_{3}\right)}{\sin (q h)}\right] C_{1}, \\
& \widehat{U}_{2}\left(X_{3}\right)=0, \\
& \widehat{U}_{3}\left(X_{3}\right)=-p\left[\frac{\sin \left(p X_{3}\right)}{\sin (p h)}-\frac{2 \kappa^{2}}{\kappa^{2}-q^{2}} \frac{\sin \left(q X_{3}\right)}{\sin (q h)}\right] C_{1},
\end{aligned}
$$

Antisymmetric Rayleigh-Lamb modes:

$$
\begin{aligned}
& \widehat{U}_{1}\left(X_{3}\right)=\mathrm{i} \kappa\left[\frac{\sin \left(p X_{3}\right)}{\cos (p h)}+\frac{2 p q}{\kappa^{2}-q^{2}} \frac{\sin \left(q X_{3}\right)}{\cos (q h)}\right] C_{2}, \\
& \widehat{U}_{2}\left(X_{3}\right)=0, \\
& \widehat{U}_{3}\left(X_{3}\right)=-p\left[\frac{\cos \left(p X_{3}\right)}{\cos (p h)}-\frac{2 \kappa^{2}}{\kappa^{2}-q^{2}} \frac{\cos \left(q X_{3}\right)}{\cos (q h)}\right] C_{2},
\end{aligned}
$$


Symmetric shear horizontal modes:

$$
\widehat{U}_{1}\left(X_{3}\right)=\widehat{U}_{3}\left(X_{3}\right)=0, \quad \widehat{U}_{2}\left(X_{3}\right)=C_{3} \cos \left(q X_{3}\right),
$$

Antisymmetric shear horizontal modes:

$$
\widehat{U}_{1}\left(X_{3}\right)=\widehat{U}_{3}\left(X_{3}\right)=0, \quad \widehat{U}_{2}\left(X_{3}\right)=C_{4} \sin \left(q X_{3}\right),
$$

where $C_{k}(k=1,2,3,4)$ are the normalization factors which are determined so that the corresponding wave field has the unit time-averaged energy flux density per unit surface area perpendicular to the $X_{1}$ direction, i.e., the following relation is satisfied:

$$
-\frac{1}{8 h} \int_{-h}^{h}\left[\overline{\hat{V}_{s}^{(\alpha)}\left(X_{3}\right)} \hat{P}_{s 1}^{\mathrm{L},(\alpha)}\left(X_{3}\right)+\widehat{V}_{s}^{(\alpha)}\left(X_{3}\right) \overline{\hat{P}_{s 1}^{\mathrm{L},(\alpha)}\left(X_{3}\right)}\right] \mathrm{d} X_{3}=1, \quad(\text { no sum on } \alpha),
$$

where "- " denotes the complex conjugate, $\widehat{V}_{s}^{(\alpha)}=-\mathrm{i} \omega^{(\alpha)} \widehat{U}_{s}^{(\alpha)}$ the particle velocity profile, and $\hat{P}_{s 1}^{\mathrm{L},(\alpha)}$ the stress profile calculated by Eq. (8a) with $U_{s}^{w}$ on the right-hand side replaced by $\widehat{U}_{s}^{(\alpha)} \exp \left[\mathrm{i} \kappa^{(\alpha)}\left(X_{1} \cos \theta^{(\alpha)}+X_{2} \sin \theta^{(\alpha)}\right)\right]$. Furthermore, $A^{(\alpha)}$ and $\theta^{(\alpha)}$ in Eq. (10) are the complex modal amplitude and the propagation angle from the $X_{1}$ axis of the corresponding mode, respectively, and $R_{j l}^{\varphi}$ represent the elements of $3 \times 3$ transformation matrix describing the counter-clockwise rotation of the coordinate axes through an angle $\varphi$ about the $X_{3}$ axis given by

$$
\left[R^{\varphi}\right]=\left[\begin{array}{ccc}
\cos \varphi & \sin \varphi & 0 \\
-\sin \varphi & \cos \varphi & 0 \\
0 & 0 & 1
\end{array}\right]
$$

\subsection{Secondary solution}

By substituting Eq. (10) into Eqs. (8b) and (9) and carrying out the cumbersome but 
straightforward calculation, the quadratic term of the stress tensor and its divergence can be written in the following forms:

$$
\begin{aligned}
& P_{j k}^{\mathrm{NL}, \mathrm{L}}\left(X_{1}, X_{2}, X_{3}, t\right)=\frac{1}{2} \tilde{P}_{j k}^{0}\left(X_{3}\right) \\
& +\frac{1}{2} \tilde{P}_{j k}^{2 \omega^{(1)}}\left(X_{3}\right) \exp \left\{2 \mathrm{i}\left[\kappa^{(1)}\left(X_{1} \cos \theta^{(1)}+X_{2} \sin \theta^{(1)}\right)-\omega^{(1)} t\right]\right\} \\
& +\frac{1}{2} \tilde{P}_{j k}^{2 \omega^{(2)}}\left(X_{3}\right) \exp \left\{2 \mathrm{i}\left[\kappa^{(2)}\left(X_{1} \cos \theta^{(2)}+X_{2} \sin \theta^{(2)}\right)-\omega^{(2)} t\right]\right\} \\
& +\frac{1}{2} \tilde{P}_{j k}^{+}\left(X_{3}\right) \exp \left\{\mathrm{i}\left[\kappa^{+}\left(X_{1} \cos \theta^{+}+X_{2} \sin \theta^{+}\right)-\left(\omega^{(1)}+\omega^{(2)}\right) t\right]\right\} \\
& +\frac{1}{2} \tilde{P}_{j k}^{-}\left(X_{3}\right) \exp \left\{\mathrm{i}\left[\kappa^{-}\left(X_{1} \cos \theta^{-}+X_{2} \sin \theta^{-}\right)-\left|\omega^{(1)}-\omega^{(2)}\right| t\right]\right\}+\text { c. c. , } \\
& j, k=1,2,3 \text {, } \\
& F_{j}^{\mathrm{NL}, \mathrm{L}}\left(X_{1}, X_{2}, X_{3}, t\right)=\frac{1}{2} \tilde{F}_{j}^{0}\left(X_{3}\right) \\
& +\frac{1}{2} \tilde{F}_{j}^{2 \omega^{(1)}}\left(X_{3}\right) \exp \left\{2 \mathrm{i}\left[\kappa^{(1)}\left(X_{1} \cos \theta^{(1)}+X_{2} \sin \theta^{(1)}\right)-\omega^{(1)} t\right]\right\} \\
& +\frac{1}{2} \tilde{F}_{j}^{2 \omega^{(2)}}\left(X_{3}\right) \exp \left\{2 \mathrm{i}\left[\kappa^{(2)}\left(X_{1} \cos \theta^{(2)}+X_{2} \sin \theta^{(2)}\right)-\omega^{(2)} t\right]\right\} \\
& +\frac{1}{2} \tilde{F}_{j}^{+}\left(X_{3}\right) \exp \left\{\mathrm{i}\left[\kappa^{+}\left(X_{1} \cos \theta^{+}+X_{2} \sin \theta^{+}\right)-\left(\omega^{(1)}+\omega^{(2)}\right) t\right]\right\} \\
& +\frac{1}{2} \tilde{F}_{j}^{-}\left(X_{3}\right) \exp \left\{\mathrm{i}\left[\kappa^{-}\left(X_{1} \cos \theta^{-}+X_{2} \sin \theta^{-}\right)-\left|\omega^{(1)}-\omega^{(2)}\right| t\right]\right\}+\text { c. c. , } \\
& j=1,2,3 \text {. }
\end{aligned}
$$

It should be noted that in the above expressions, c.c. represents the complex conjugate of the sum of the preceding terms. In Eq. (16), $\kappa^{ \pm}$and $\theta^{ \pm}$are given by 


$$
\begin{aligned}
& \kappa^{ \pm}=\sqrt{\left[\kappa^{(1)} \cos \theta^{(1)} \pm \kappa^{(2)} \cos \theta^{(2)}\right]^{2}+\left[\kappa^{(1)} \sin \theta^{(1)} \pm \kappa^{(2)} \sin \theta^{(2)}\right]^{2}} \\
& \cos \theta^{ \pm}=\frac{\kappa^{(1)} \cos \theta^{(1)} \pm \kappa^{(2)} \cos \theta^{(2)}}{\kappa^{ \pm}} G\left(\omega^{(1)} \pm \omega^{(2)}\right) \\
& \sin \theta^{ \pm}=\frac{\kappa^{(1)} \sin \theta^{(1)} \pm \kappa^{(2)} \sin \theta^{(2)}}{\kappa^{ \pm}} G\left(\omega^{(1)} \pm \omega^{(2)}\right)
\end{aligned}
$$

where the double sign applies in the same order and

$$
G(\xi) \equiv\left\{\begin{array}{rr}
1, & \xi \geq 0 \\
-1, & \xi<0
\end{array}\right.
$$

The coefficients $\tilde{P}_{j k}^{M}$ and $\tilde{F}_{j}^{M}\left(M=0,2 \omega^{(1)}, 2 \omega^{(2)},+\right.$, and -$)$ in Eq. (16) represent the thickness distributions of the driving forces for the nonlinear interaction, but their expressions are too lengthy to be described in this paper. Equation (16) clearly indicates that the driving terms for the secondary wave field consist of the DC component $(\omega=0)$, the double-frequency components $\left(\omega=2 \omega^{(1)}\right.$ and $\left.\omega=2 \omega^{(2)}\right)$ due to the self-interaction of each primary mode, and the sum- and difference-frequency components $\left(\omega=\omega^{(1)}+\right.$ $\omega^{(2)}$ and $\left.\omega=\left|\omega^{(1)}-\omega^{(2)}\right|\right)$ due to the mutual interaction of two primary modes. Since the present interest is in examining the generation behavior of secondary waves due to the non-collinear interaction, Eq. (16) is rewritten with the DC and the second-harmonic terms omitted as follows:

$$
P_{j k}^{\mathrm{NL}, \mathrm{L}}=\frac{1}{2} \tilde{P}_{j k}^{ \pm}\left(X_{3}\right) \exp \left\{\mathrm{i}\left[\kappa^{ \pm}\left(X_{1} \cos \theta^{ \pm}+X_{2} \sin \theta^{ \pm}\right)-\omega^{ \pm} t\right]\right\}+\text { c. c. }
$$




$$
F_{j}^{\mathrm{NL}, \mathrm{L}}=\frac{1}{2} \tilde{F}_{j}^{ \pm}\left(X_{3}\right) \exp \left\{\mathrm{i}\left[\kappa^{ \pm}\left(X_{1} \cos \theta^{ \pm}+X_{2} \sin \theta^{ \pm}\right)-\omega^{ \pm} t\right]\right\}+\text { c. c. , }
$$

where $\omega^{ \pm}=\left|\omega^{(1)} \pm \omega^{(2)}\right|$ and the superscripts "+" and "-" correspond to the sum- and difference-frequency components, respectively.

Equation (19) indicates that the secondary wave field can be expressed as a superposition of plate wave modes propagating in the $\theta^{ \pm}$direction. By introducing a new coordinate system $X_{1}{ }^{\prime}-X_{2}{ }^{\prime}-X_{3}{ }^{\prime}$ which is given by rotating the $X_{1}-X_{2}-X_{3}$ system through an angle $\theta^{ \pm}$about the $X_{3}$ axis as shown in Fig. $1\left(X_{3}{ }^{\prime}=X_{3}\right)$, the $X_{j}{ }^{\prime}$ components of the secondary solution, denoted by $U_{j}^{\prime \mathrm{NL}}$, are written as

$$
U_{j}^{\prime \mathrm{NL}}\left(X_{1}^{\prime}, X_{2}^{\prime}, X_{3}^{\prime}, t\right)=\frac{1}{2} \sum_{m=1}^{\infty} A_{m}\left(X_{1}^{\prime}\right) \widehat{U}_{j}^{\prime(m)}\left(X_{3}^{\prime}\right) \exp \left(-\mathrm{i} \omega^{ \pm} t\right)+\text { c. c. }, \quad j=1,2,3
$$

where $\widehat{U}_{j}^{\prime(m)}$ represent the $X_{j}^{\prime}$ components of normalized displacement profile of the $m$ th plate wave mode with the wavenumber $\kappa_{m}$ and the angular frequency $\omega^{ \pm}$ propagating in the $X_{1}{ }^{\prime}$ direction, whose expressions are given by Eq. (13) with $p$ and $q$ given by Eq. (12) with $\omega=\omega^{ \pm}$and $\kappa=\kappa_{m}$. Note that when the wave mode is the propagating mode, the displacement profile is normalized in accordance with Eq. (14) as in the case of the primary waves. On the other hand, when the wave mode becomes evanescent with $\operatorname{Im}\left(\kappa_{m}\right) \neq 0$, the normalization based on Eq. (14) is inapplicable since the corresponding mode has no power flow. Hence, $\widehat{U}_{j}^{\prime(m)}$ of such modes are assumed to be normalized so that the following relation is satisfied instead of Eq. (14) [40]: 


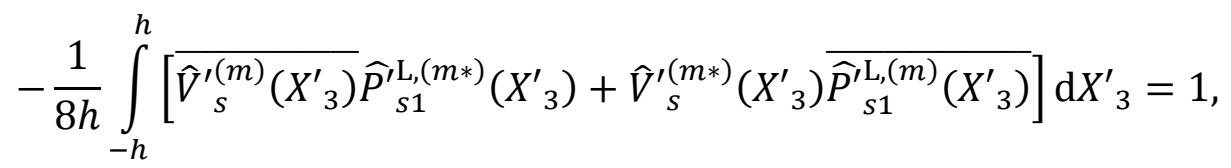

where $\widehat{V}_{s}^{\prime(m)}=-\mathrm{i} \omega^{ \pm} \widehat{U}_{s}^{\prime(m)}\left(\widehat{P}_{s 1}^{\mathrm{L},(m)}\right)$ and $\widehat{V}_{s}^{\prime(m *)}=-\mathrm{i} \omega^{ \pm} \widehat{U}_{s}^{\prime(m *)}\left(\widehat{P}_{s 1}^{\mathrm{L},(m *)}\right)$ represent the particle velocity (stress) profile of the wave modes with the wavenumber $\kappa_{m}$ and $\overline{\kappa_{m}}$, respectively.

Assuming that the displacements of secondary wave are zero at $X_{1}{ }^{\prime}=0$, the modal amplitude $A_{m}\left(X_{1}^{\prime}\right)$ in Eq. (20) is given by making use of the orthogonality of the plate wave modes as $[21,32,33,40]$,

$$
A_{m}\left(X_{1}^{\prime}\right)=\frac{f_{m}^{\text {surf }}+f_{m}^{\text {vol }}}{2 h} X_{1}^{\prime} \operatorname{sinc}\left(\frac{\kappa^{ \pm}-\kappa_{m}}{2} X_{1}^{\prime}\right) \exp \left(\mathrm{i} \frac{\kappa^{ \pm}+\kappa_{m}}{2} X_{1}^{\prime}\right)
$$

where

$$
\begin{gathered}
f_{m}^{\text {surf }}=-\left.\frac{1}{4} \overline{\widehat{V}_{s}^{(m *)}\left(X_{3}^{\prime}\right)} \tilde{P}_{s 3}^{\prime \pm}\left(X_{3}^{\prime}\right)\right|_{X_{3}^{\prime}=-h^{\prime}} ^{X_{3}^{\prime}=h}, \\
f_{m}^{\text {vol }}=\frac{1}{4} \int_{-h}^{h} \overline{\widehat{V}_{s}^{(m *)}\left(X_{3}^{\prime}\right)} \tilde{F}_{s}^{\prime \pm}\left(X_{3}^{\prime}\right) \mathrm{d} X_{3}^{\prime},
\end{gathered}
$$

represent the power flux through the surface and volume of the plate due to the primary wave. In Eq. (23),

$$
\begin{aligned}
& \tilde{P}_{s 3}^{\prime \pm}\left(X_{3}^{\prime}\right)=R_{s r}^{\theta^{ \pm}} \tilde{P}_{r l}^{ \pm}\left(X_{3}^{\prime}\right) R_{l 3}^{-\theta^{ \pm}}=R_{s r}^{\theta^{ \pm}} \tilde{P}_{r 3}^{ \pm}\left(X_{3}^{\prime}\right), \\
& \tilde{F}_{s}^{\prime \pm}\left(X_{3}^{\prime}\right)=R_{s r}^{\theta^{ \pm}} \tilde{F}_{r}^{ \pm}\left(X_{3}^{\prime}\right), \quad s=1,2,3,
\end{aligned}
$$


where the double sign applies in the same order. The secondary solution expressed in the original $X_{1}-X_{2}-X_{3}$ coordinate system can be given as $U_{j}^{\mathrm{NL}}=R_{j r}^{-\theta^{ \pm}} U_{r}^{\prime \mathrm{NL}}(j=1,2,3)$.

From Eq. (22), the modal amplitude grows in proportion with the distance in the $X_{1}{ }^{\prime}$ direction if $f_{m}^{\text {surf }}+f_{m}^{\mathrm{vol}} \neq 0$ and the wavevector of the primary modes and the plate wave mode used in the expansion of the secondary solution, denoted by $\boldsymbol{\kappa}^{(\alpha)}=\left(\kappa^{(\alpha)} \cos \right.$ $\left.\theta^{(\alpha)}, \kappa^{(\alpha)} \sin \theta^{(\alpha)}, 0\right)(\alpha=1,2)$ and $\boldsymbol{\kappa}_{m}=\left(\kappa_{m} \cos \theta^{ \pm}, \kappa_{m} \sin \theta^{ \pm}, 0\right)$, respectively, satisfies

$$
\boldsymbol{\kappa}_{m}=\boldsymbol{\kappa}^{(1)} \pm \boldsymbol{\kappa}^{(2)}
$$

The above relation is equivalent to the so-called resonance condition for the occurrence of non-collinear resonant interaction of bulk elastic waves [4]. Note that Eq. (25) includes the condition for the collinear resonant interaction of plate wave modes derived by de Lima and Hamilton [21] as a special case for $\theta^{(1)}=\theta^{(2)}=0$. Furthermore, Eq. (25) can be satisfied only when the secondary wave mode is the propagating mode with $\operatorname{Im}\left(\kappa_{m}\right)=0$ since the primary waves are assumed to be the propagating modes with $\operatorname{Im}\left(\kappa^{(\alpha)}\right)=0$ in the present study.

\subsection{Symmetry property of the secondary wave mode}

When the primary wave modes are both symmetric or both antisymmetric, $\tilde{P}^{\prime t} s 3$ and $\tilde{F}_{\frac{S}{s}}^{\prime \pm}$ in Eq. (24) have the following symmetric properties irrespective of their propagation angles $\theta^{(\alpha)}$ :

$$
\left(\begin{array}{c}
\tilde{P}_{13}^{\prime \pm} \\
\tilde{P}_{23}^{\prime \pm} \\
\tilde{P}_{33}^{\prime \pm}
\end{array}\right):\left(\begin{array}{l}
\mathrm{A} \\
\mathrm{A} \\
\mathrm{S}
\end{array}\right), \quad\left(\begin{array}{c}
\tilde{F}_{1}^{\prime \pm} \\
\tilde{F}^{\prime \pm} \\
\tilde{F}^{\prime \frac{ \pm}{3}}
\end{array}\right):\left(\begin{array}{c}
\mathrm{S} \\
\mathrm{S} \\
\mathrm{A}
\end{array}\right)
$$


where " $\mathrm{S}$ " and " $\mathrm{A}$ " denote that the corresponding component is symmetric and antisymmetric with respect to $X_{3}{ }^{\prime}=0$, respectively. This indicates from Eqs. (13b), (13d), and (23) that $f_{m}^{\text {surf }}=f_{m}^{\text {vol }}=0$ for all antisymmetric modes used in the expansion of the secondary wave field. Therefore, the interaction of two symmetric or two antisymmetric modes can produce only the symmetric modes. In contrast, when the primary wave consists of a pair of symmetric and antisymmetric modes, $\tilde{P}_{s 3}^{\prime \pm}$ and $\tilde{F}^{\prime \pm}$ become

$$
\left(\begin{array}{c}
\tilde{P}_{13}^{\prime \pm} \\
\tilde{P}^{\prime \frac{ \pm}{23}} \\
\tilde{P}_{33}^{\prime \pm}
\end{array}\right):\left(\begin{array}{l}
\mathrm{S} \\
\mathrm{S} \\
\mathrm{A}
\end{array}\right), \quad\left(\begin{array}{c}
\tilde{F}^{\prime \pm} \\
\tilde{F}^{\prime \pm} \\
\tilde{F}^{\prime \pm}
\end{array}\right):\left(\begin{array}{c}
\mathrm{A} \\
\mathrm{A} \\
\mathrm{S}
\end{array}\right) .
$$

The resulting secondary wave field, hence, consists of only the antisymmetric modes from Eqs. (13a), (13c), and (23).

\section{Results and discussions for the interaction of lowest-order modes}

In this section, the generation behavior of secondary wave field due to the non-collinear interaction in an aluminum alloy plate is examined. The elastic properties of the plate are shown in Table 1. Note that Eq. (22) can be applied to calculate the modal amplitude of evanescent modes, while the present study deals only with the propagating modes in the secondary wave field.

The dispersion relation of plate wave modes in Eq. (11) is shown for a lower frequency range in Fig. 2, where the horizontal and vertical axes represent the non-dimensional wavenumber $K=2 \kappa h$ and the non-dimensional frequency $\Omega=$ $2 \omega h /\left(\pi c_{\mathrm{T}}\right)$, respectively. The analysis below focuses on a low-frequency range where the lowest-order symmetric and antisymmetric Rayleigh-Lamb waves (RLS0 and RLA0) 
and the lowest-order symmetric shear horizontal wave (SHSO) are the only propagating modes for the primary as well as the secondary wave fields. Specifically, the non-dimensional frequencies of two primary modes, denoted by $\Omega^{(\alpha)}=2 \omega^{(\alpha)} h /\left(\pi c_{\mathrm{T}}\right)(\alpha=$ $1,2)$, are assumed to be

$$
\left\{\Omega^{(1)}, \Omega^{(2)}, \Omega^{(1)}+\Omega^{(2)},\left|\Omega^{(1)}-\Omega^{(2)}\right|\right\} \subset(0,1),
$$

where $\Omega=1$ corresponds to the cut-off frequency of the first-order antisymmetric Rayleigh-Lamb (RLA1) as well as the lowest-order antisymmetric shear horizontal (SHA0) modes.

\subsection{Intersection angle for the resonant interaction}

For the frequency range given in Eq. (28), the existence of intersection angles which satisfy the resonance condition in Eq. (25) is summarized in Table 2. The secondary modes which are not generated because of the symmetry relation mentioned in Section 3.3 are labeled "NG." Besides these forbidden modes, the generation of the SHS0 mode due to the interaction of two SHS0 modes is also impossible and labeled "NG" since $\tilde{P}^{\prime \pm}{ }_{23}=\tilde{F}^{\prime \pm}=0$ is always satisfied, i.e., $f_{m}^{\text {surf }}=f_{m}^{\text {vol }}=0$ for this mode. For the other cases, the existence of the intersection angle satisfying the resonance condition is examined by seeking for a numerical solution of Eq. (25) for the assumed elastic parameters. In Table 2, the label "R/NR" indicates that such angles are found for a certain range of primary frequencies, and "NR" that no intersection angles satisfying the resonance condition are found for any combinations of primary frequencies. It is noted that the existence of the resonant angle depends on the linear dispersion relations of the plate in Eq. (11), and determined by Poisson's ratio of the plate. Therefore, the classification of "R/NR" and "NR" in Table 2 is for the specific material parameters 
assumed here, while the label "NG" holds irrespective of them.

From Table 2, five combinations of primary and secondary wave modes have resonant angles for the sum-frequency component, and six combinations do for the difference-frequency component. As a general trend, these angles exist when the phase velocity of the secondary mode with the sum- and difference-frequencies is faster and slower than the primary modes, respectively. The variations of these angles with the primary frequencies are depicted in Figs. 3 and 4. Likewise, the frequency dependence of resonant angles for the non-collinear interaction of bulk longitudinal and shear waves [4], denoted by "L" and "T", respectively, is shown for comparison in Fig. 5. Note that there is essentially no need to restrict the frequency for the interaction of bulk waves without cut-off frequencies, but the results are depicted in Fig. 5 only for the frequency range given by Eq. (28) in order to compare with the interaction of guided waves in Figs. 3 and 4. Furthermore, it appears in Figs. 3-5 that the numerically obtained resonant angles for some mode combinations have their limits for the null primary frequencies, such as for $\Omega^{(2)} \rightarrow 0$ in Figs. 3(d), 4(c), 5(b), and 5(d). In these cases, however, the sumand difference-frequency components are not generated since both $P_{j k}^{\mathrm{NL}, \mathrm{L}}$ and $F_{j}^{\mathrm{NL}, \mathrm{L}}$ in Eq. (19) vanish.

For the bulk wave interaction in an isotropic solid in Fig. 5, two and three interaction cases have resonant angles for the sum- and difference-frequency components, respectively, and they are governed by the ratio of primary frequencies [4]. In contrast, those for the plate wave interaction in Figs. 3 and 4 are influenced by the magnitude of two primary frequencies due to their dispersive nature. Comparing Figs. 3 and 4 with Fig. 5, the resonant angles for the interaction of RLS0 and SHS0 modes in Figs. 3(a), 3(d), 4(a), 4(c), and 4(d) exhibit the frequency dependence similar to that for 
the interaction of $\mathrm{L}$ and $\mathrm{T}$ modes in Figs. 5(a)-5(e). This is because of the weak dispersion of RLS0 mode in the present low-frequency range and the non-dispersive nature of SHS0 mode. On the other hand, it can be clearly seen in Figs. 3(b), 3(c), 3(e), 4(b), and 4(f) that the resonant angles for the interaction involving the RLA0 mode vary markedly with the magnitude of the primary frequencies due to its more significant dispersive nature.

Most of the mode combinations in Figs. 3 and 4 have a wide range of resonant angles depending on the primary frequencies. When the primary modes are SHSO and RLA0 in Fig. 3(e) and when they are both RLA0 in Fig. 4(b), however, the resonant interaction is localized at very small intersection angles of less than $10^{\circ}$ and $5^{\circ}$, respectively.

\subsection{Modal amplitude of secondary wave field}

Based on the results for the resonant angles mentioned above in Section 4.1, the generation behavior of secondary wave due to the non-collinear interaction is examined below in more detail for some representative combinations of primary plate wave modes.

The modal amplitudes of the sum- and difference-frequency components of RLS0 and SHS0 modes generated by the RLS0-SHS0 interaction are calculated by Eq. (22) for fixed primary frequencies (wavenumbers) of $\Omega^{(1)}=0.5\left(K^{(1)}=2 \kappa^{(1)} h=0.91\right)$ and $\Omega^{(2)}$ $=0.2\left(K^{(2)}=2 \kappa^{(2)} h=0.63\right)$ for the RLS0 and SHS0 modes, respectively. Their variation with the intersection angle is shown for different propagation distances in Fig. 6, together with the propagation angle as well as the wavenumber of the secondary wave.

From Eqs. (17b) and (17c) with $\kappa^{(1)}>\kappa^{(2)}$ and $\omega^{(1)}>\omega^{(2)}$, the propagation angle of secondary wave from the first primary mode shown in Fig. 6(a) becomes positive and 
negative for the sum- and difference-frequency components, respectively. The horizontal dotted and chain lines in Fig. 6(b) represent the wavenumber of the plate wave modes used in the expansion of the secondary wave; namely, the intersection angles where the curves of $2 \kappa^{ \pm} h$ cross these lines correspond to the resonant angles. For the sum-frequency component, the resonant angle for the RLS0 mode is $67^{\circ}$ and the one for the SHS0 mode does not exist. Likewise, the resonant angles for the RLS0 and SHS0 modes are $36^{\circ}$ and $73^{\circ}$, respectively, for the difference-frequency component. These angles are indicated by vertical lines in Figs. 6(c), 6(d), and 6(f). It is seen in Figs. 6(c), 6(d), and 6(f) that the modal amplitudes exhibit the monotonic growth with the propagation distance at around the resonant angles, while such a trend is not seen in the absence of the resonant angle in Fig. 6(e).

The corresponding results for the SHS0-RLA0 interaction are shown in Fig. 7, where the primary frequencies (wavenumbers) are $\Omega^{(1)}=0.2\left(K^{(1)}=0.63\right)$ for the SHS0 mode and $\Omega^{(2)}=0.7\left(K^{(2)}=2.9\right)$ for the RLA0 mode. From Eqs. $(17 \mathrm{~b})$ and $(17 \mathrm{c})$ with $\kappa^{(1)}<\kappa^{(2)}$ and $\omega^{(1)}<\omega^{(2)}$, the propagation angles of both sum- and difference-frequency components in Fig. 7(a) become positive and increase monotonically from $0^{\circ}$ to $180^{\circ}$ with the intersection angle. As shown in Table 2, the only propagating mode produced by this interaction is the RLA0 mode, for which the resonant angles of the sum- and difference-frequency components are $9.6^{\circ}$ and $3.1^{\circ}$, respectively, from Fig. 7(b). It is found in Figs. 7(c) and 7(d) that the modal amplitudes grow with the propagation distance near the resonant angles as in the case of the RLS0-SHS0 interaction in Fig. 6, while the intersection angles for the maximum amplitude deviate from the resonant angles. This trend was also presented by Matsuda and Biwa [33] regarding the frequency dependence of the second-harmonic generation of a monochromatic 
Rayleigh-Lamb wave. These authors showed that the second-harmonic amplitude becomes maximal for the frequency deviated from the one satisfying the phase-matching condition between the fundamental and its second-harmonic modes. The reason for such behavior is that the amplitude of secondary wave is governed by not only the resonance condition but also the factors representing the power flux from the primary to the secondary waves, $f_{m}^{\text {surf }}$ and $f_{m}^{\text {vol }}$ [33]. As can be seen in Figs. 7(c) and $7(\mathrm{~d})$, the locations of peak amplitude approach the resonant angles as the propagation distance increases, since the influence of resonance condition becomes dominant for larger propagation distance as represented by the cardinal sine function in Eq. (22). The contribution of the resonance condition also depends on the choice of the primary wave modes. In contrast to the SHS0-RLA0 interaction in Fig. 7, the RLS0-SHS0 interaction in Fig. 6 shows negligible deviation of the maximum amplitude angle from the resonant angle.

For the mode combinations shown in Figs. 3 and 4, the modal amplitude is calculated with various intersection angles $\theta^{(2)}-\theta^{(1)}$ and the second primary frequencies $\Omega^{(2)}$ when the first primary frequency and the propagation distance are fixed as $\Omega^{(1)}=$ 0.5 and $X_{1}{ }^{\prime} / h=100$, respectively. The results are illustrated in Figs. 8 and 9, together with the resonance conditions by dotted lines.

As mentioned above in Fig. 7, the modal amplitude in Figs. 8(e), 9(b), and Fig. 9(f) (for $\Omega^{(2)}>0.5$ ) becomes large for the intersection angle and the primary frequencies away from the resonance condition due to the short propagation distance. For the other interaction cases in Figs. 8(a)-8(d), 9(a), and 9(c)-9(e), the locations of large amplitude agree well with the resonance conditions, except that the modal amplitude becomes vanishingly small in certain ranges even when the resonance condition is satisfied, such 
as at around $\left(\theta^{(2)}-\theta^{(1)}, \Omega^{(2)}\right)=\left(139^{\circ}, 0.15\right)$ in Fig. $8($ a $)$ and $\left(\theta^{(2)}-\theta^{(1)}, \Omega^{(2)}\right)=\left(90^{\circ}, 0.2\right)$

in Fig. 9(a). This is due to the fact that $f_{m}^{\text {surf }}+f_{m}^{\text {vol }}$ becomes vanishingly small therein: for example, in the case of the SHS0-SHS0 interaction producing the RLS0 mode in Fig. 8(a), the straightforward calculation of Eq. (23) results in that $f_{m}^{\text {surf }}=0$ when

$$
\tan \left[\theta^{(2)}-\theta^{(1)}\right]= \pm \sqrt{\frac{\lambda+\mathcal{B}}{\mathcal{B}}}
$$

and $f_{m}^{\mathrm{vol}}=0$ when

$$
\tan \left[\theta^{(2)}-\theta^{(1)}\right]= \pm \sqrt{\frac{2 \lambda+4 \mu+\mathcal{A}+2 \mathcal{B}}{2 \mu+\mathcal{A}+2 \mathcal{B}}}
$$

Using the elastic properties in Table 1 and assuming that $0 \leq \theta^{(2)}-\theta^{(1)} \leq 180^{\circ}, f_{m}^{\text {surf }}$ vanishes at $\theta^{(2)}-\theta^{(1)}=40.2^{\circ}$ and $139.8^{\circ}$, and $f_{m}^{\text {vol }}$ does at $\theta^{(2)}-\theta^{(1)}=41.4^{\circ}$ and $138.6^{\circ}$, so $f_{m}^{\text {surf }}+f_{m}^{\text {vol }}$ drops to very low levels at around $\theta^{(2)}-\theta^{(1)}=139^{\circ}$. Note that the intersection angles for $f_{m}^{\text {surf }}=0$ and $f_{m}^{\text {vol }}=0$ in Eqs. (29) and (30) are independent of the frequency, while those for the other mode combinations are influenced by not only the elastic constants but also the primary frequencies.

\section{Conclusions}

Non-collinear interaction of guided elastic waves in an isotropic plate has been analyzed theoretically in this paper. Using the perturbation analysis, an explicit expression for the modal amplitude of the secondary wave having the sum or difference frequency of the primary waves has been derived, and the resulting amplitude has been shown to increase linearly with the propagation distance when the resonance condition 
is satisfied. It has been shown that the interaction of two symmetric or two antisymmetric modes produces the symmetric modes only, while a pair of symmetric and antisymmetric primary modes does the antisymmetric modes only. The modal amplitude has been calculated for various intersection angles, primary frequencies, and mode combinations for a low-frequency range where the lowest-order symmetric and antisymmetric Rayleigh-Lamb waves and the lowest-order symmetric shear horizontal wave are the only propagating modes. It has been shown that the modal amplitude for short propagation distance can be maximal at the intersection angles deviated from the resonant angles. Furthermore, the modal amplitude can be very small even when the resonance condition is satisfied if the power flux from the primary to the secondary wave is small.

In the case of the second-harmonic generation of Rayleigh-Lamb waves in an isotropic plate, the phase matching between the fundamental and its second-harmonic modes for the cumulative growth of the second harmonics with the propagation distance is satisfied only above the cut-off frequencies of the lowest-order modes [20,29,31]. On the other hand, the resonance condition for the non-collinear interaction can be met even below these cut-off frequencies by selecting the appropriate primary frequencies and intersection angles as shown in Section 4.2. This is practically an important feature in order to measure the nonlinear effect in plate-like structures with their complicated multimode nature suppressed.

This paper assumed the plane primary waves with infinite beam widths as a fundamental study to investigate the non-collinear interaction in plates. From a practical point of view, the interaction of plate waves with finite beam widths is an important issue to be explored. In this situation, the cumulative growth of secondary waves with 
the propagation distance presented in this paper is expected to occur only in a limited volume where the two primary waves interact. In the case of non-collinear interaction of bulk waves $[4,7]$, the resonance condition derived on the basis of plane-wave theory was shown to be still valid for the primary waves with finite beam widths, and the amplitude of secondary waves propagating in the direction determined by this condition was shown to increase linearly with the volume of interaction of two primary waves. In light of these features, the resonance condition derived in the present study can be expected to play an important role even in the interaction of plate waves with finite beam widths. This issue can be investigated by extending the analysis presented in this paper to a finite region of intersection as in Refs. [4] and [7], which is left for the future work. Furthermore, this paper dealt with the non-collinear interaction of monochromatic plate waves, while the transient response when mixing pulse waves is another issue to be investigated by, for example, performing numerical simulations.

\section{Acknowledgements}

This work has been supported by JSPS KAKENHI Grant Numbers JP17K14557 and JP16H04235. 


\section{References}

[1] M.A. Breazeale, J. Ford, Ultrasonic studies of the nonlinear behavior of solids, J. Appl. Phys. 36 (1965) pp. 3486-3490.

[2] R.B. Thompson, O. Buck, D.O. Thompson, Higher harmonics of finite amplitude ultrasonic waves in solids, J. Acoust. Soc. Am. 59 (1976) pp. 1087-1094.

[3] K.H. Matlack, J.-Y. Kim, L.J. Jacobs, J. Qu, Review of second harmonic generation measurement techniques for material state determination in metals, J. Nondestruct. Eval. 34: 273 (2015) pp. 1-23.

[4] G.L. Jones, D.R. Kobett, Interaction of elastic waves in an isotropic solid, J. Acoust. Soc. Am. 35 (1963) pp. 5-10.

[5] L.H. Taylor, F.R. Rollins Jr., Ultrasonic study of three-phonon interactions. I. Theory, Phys. Rev. 136 (1964) pp. A591-A596.

[6] F.R. Rollins Jr., Phonon interactions at ultrasonic frequencies, Proc. of the IEEE 53 (1965) pp. 1534-1539.

[7] I.A. Beresnev, Interaction of two spherical elastic waves in a nonlinear five-constant medium, J. Acoust. Soc. Am. 94 (1993) pp. 3400-3404.

[8] V.A. Korneev, A. Demčenko, Possible second-order nonlinear interactions of plane waves in an elastic solid, J. Acoust. Soc. Am. 135 (2014) pp. 591-598.

[9] A. Demčenko, L. Mainini, V.A. Korneev, A study of the noncollinear ultrasonic-wave-mixing technique under imperfect resonance conditions, Ultrasonics 57 (2015) pp. 179-189.

[10]Z. Sun, F. Li, H. Li, A numerical study of non-collinear wave mixing and generated resonant components, Ultrasonics 71 (2016) pp. 245-255.

[11]F.R. Rollins Jr., L.H. Taylor, P.H. Todd Jr., Ultrasonic study of three-phonon 
interactions. II. Experimental results, Phys. Rev. 136 (1964) pp. A597-A601.

[12] M. Hirao, A. Tomizawa, H. Fukuoka, Nonlinear resonance interaction of ultrasonic waves under applied stress, J. Appl. Phys. 56 (1984) pp. 235-237.

[13]A.J. Croxford, P.D. Wilcox, B.W. Drinkwater, The use of non-collinear mixing for nonlinear ultrasonic detection of plasticity and fatigue, J. Acoust. Soc. Am. 126 (2009) pp. EL117-EL122.

[14]A. Demčenko, R. Akkerman, P.B. Nagy, R. Loendersloot, Non-collinear wave mixing for non-linear ultrasonic detection of physical ageing in PVC, NDT\&E Int. 49 (2012) pp. 34-39.

[15]A. Demčenko, V. Koissin, V.A. Korneev, Noncollinear wave mixing for measurement of dynamic processes in polymers: Physical ageing in thermoplastics and epoxy cure, Ultrasonics 54 (2014) pp. 684-693.

[16]M.E. McGovern, W.G. Buttlar, H. Reis, Characterisation of oxidative ageing in asphalt concrete using a non-collinear ultrasonic wave mixing approach, Insight 56 (2014) pp. 367-374.

[17]P. Blanloeuil, A.J. Croxford, A. Meziane, Application of the noncollinear mixing method to an interface of contact, Proceedings of the $40^{\text {th }}$ Annual Review of Progress in Quantitative Nondestructive Evaluation (2013) pp. 623-630.

[18]Z. Zhang, P.B. Nagy, W. Hassan, Analytical and numerical modeling of non-collinear shear wave mixing at imperfect interface, Ultrasonics 65 (2016) pp. 165-176.

[19] M. Deng, Cumulative second-harmonic generation of Lamb-mode propagation in a solid plate, J. Appl. Phys. 85 (1999) pp. 3051-3058.

[20] M. Deng, Analysis of second-harmonic generation of Lamb modes using a modal 
analysis approach, J. Appl. Phys. 94 (2003) pp. 4152-4159.

[21] W.J.N. de Lima, M.F. Hamilton, Finite-amplitude wave in isotropic elastic plates, J. Sound Vib. 265 (2003) pp. 819-839.

[22] M. Deng, P. Wang, X. Lv, Experimental observation of cumulative second-harmonic generation of Lamb-wave propagation in an elastic plate, J. Phys. D 38 (2005) pp. 344-353.

[23] M. Deng, J. Pei, Assessment of accumulated fatigue damage in solid plates using nonlinear Lamb wave approach, Appl. Phys. Lett. 90 (2007) 121902.

[24] C. Pruell, J.-Y. Kim, J. Qu, L.J. Jacobs, Evaluation of plasticity driven material damage using Lamb waves, Appl. Phys. Lett. 91 (2007) 231911.

[25] C. Bermes, J.-Y. Kim, J. Qu, L.J. Jacobs, Experimental characterization of material nonlinearity using Lamb waves, Appl. Phys. Lett. 90 (2007) 021901.

[26] A. Srivastava, F. Lanza di Scalea, On the existence of antisymmetric or symmetric Lamb waves at nonlinear higher harmonics, J. Sound Vib. 323 (2009) pp. 932-943.

[27] Y. Xiang, M. Deng, F.-Z. Xuan, C.-J. Liu, Experimental study of thermal degradation in ferritic $\mathrm{Cr}-\mathrm{Ni}$ alloy steel plates using nonlinear Lamb waves, NDT\&E Int. 44 (2011) pp. 768-774.

[28] Y. Xiang, M. Deng, F.-Z. Xuan, Analysis of second-harmonic generation of Lamb waves using a combined method in a two layered solid waveguide, J. Appl. Phys. $106(2009) 024902$.

[29] M.F. Müller, J.-Y. Kim, J. Qu, L.J. Jacobs, Characteristics of second harmonic generation of Lamb waves in nonlinear elastic plates, J. Acoust. Soc. Am. 127 (2010) pp. 2141-2152.

[30] Y. Xiang, M. Deng, F.-Z. Xuan, C.-J. Liu, Cumulative second-harmonic analysis of 
ultrasonic Lamb waves for ageing behavior study of modified-HP austenite steel, Ultrasonics 51 (2011) pp. 974-981.

[31] N. Matsuda, S. Biwa, Phase and group velocity matching for cumulative harmonic generation in Lamb waves, J. Appl. Phys. 109 (2011) 094903.

[32] Y. Liu, V.K. Chillara, C.J. Lissenden, On selection of primary modes for generation of strong internally resonant second harmonics in plate, J. Sound Vib. 332 (2013) pp. $4517-4528$.

[33]N. Matsuda, S. Biwa, Frequency dependence of second-harmonic generation in Lamb waves, J. Nondestruct. Eval. 33 (2014) pp. 169-177.

[34]Z. Peng, Z. Yu, F. Zheng, Numerical and experimental investigation of nonlinear ultrasonic Lamb waves at low frequency, Appl. Phys. Lett. 109 (2016) 021902.

[35] J. Jiao, X. Meng, C. He, B. Wu, Nonlinear Lamb wave-mixing technique for micro-crack detection in plates, NDT\&E Int. 85 (2017) pp. 63-71.

[36] C.J. Lissenden, Y. Liu, V.K. Chillara, G. Choi, H. Cho, Nonlinear guided wave mixing for localized material state characterization, Physics Procedia 70 (2015) pp. 668-671.

[37] E.S. Furgason, V.L. Newhouse, Noncollinear three-phonon interactions in a multimode structure, J. Appl. Phys. 45 (1974) pp. 1934-1936.

[38] L.D. Landau, E.M. Lifshitz, Theory of elasticity, Pergamon, New York (1959).

[39] A.N. Norris, Finite-amplitude waves in solids, in: M.F. Hamilton, D.T. Blackstock (Eds.), Nonlinear Acoustics, Academic Press, New York, 1998, pp. 263-277.

[40] B.A. Auld, Acoustic field and waves in solids, Wiley, London, 1973.

[41] J.L. Rose, Ultrasonic waves in Solid media, Cambridge University Press, London, 1975. 
[42] R.T. Smith, R. Stern, R.W.B. Stephens, Third-order elastic moduli of polycrystalline metals from ultrasonic velocity measurements, J. Acoust. Soc. Am. 40 (1966) pp. 1002-1008. 
Table 1 Material properties of the aluminum alloy plate [42].

\begin{tabular}{cccccc}
\hline$\rho_{0}\left(\mathrm{~kg} / \mathrm{m}^{3}\right)$ & $\lambda(\mathrm{GPa})$ & $\mu(\mathrm{GPa})$ & $\mathcal{A}(\mathrm{GPa})$ & $\mathcal{B}(\mathrm{GPa})$ & $\mathcal{C}(\mathrm{GPa})$ \\
\hline 2,700 & 56.0 & 26.5 & -408 & -197 & -114 \\
\hline
\end{tabular}

Table 2 Existence of intersection angles for the non-collinear resonant interaction of lowest-order plate modes ${ }^{\mathrm{a}}$.

Secondary modes

\begin{tabular}{ccccccc}
\cline { 2 - 6 } Pair of & \multicolumn{2}{c}{ RLS0 } & \multicolumn{2}{c}{ SHS0 } & \multicolumn{2}{c}{ RLA0 } \\
\cline { 2 - 7 } primary modes & $\Omega^{(1)}+\Omega^{(2)}\left|\Omega^{(1)}-\Omega^{(2)}\right|$ & $\Omega^{(1)}+\Omega^{(2)}$ & $\left|\Omega^{(1)}-\Omega^{(2)}\right|$ & $\Omega^{(1)}+\Omega^{(2)}\left|\Omega^{(1)}-\Omega^{(2)}\right|$ \\
\hline RLS0 \& RLS0 & $\mathrm{NR}$ & $\mathrm{NR}$ & $\mathrm{NR}$ & $\mathrm{R} / \mathrm{NR}$ & $\mathrm{NG}$ & $\mathrm{NG}$ \\
\hline SHS0 \& SHS0 & $\mathrm{R} / \mathrm{NR}$ & $\mathrm{NR}$ & $\mathrm{NG}$ & $\mathrm{NG}$ & $\mathrm{NG}$ & $\mathrm{NG}$ \\
\hline RLA0 \& RLA0 & $\mathrm{R} / \mathrm{NR}$ & $\mathrm{NR}$ & $\mathrm{R} / \mathrm{NR}$ & $\mathrm{R} / \mathrm{NR}$ & $\mathrm{NG}$ & $\mathrm{NG}$ \\
\hline RLS0 \& SHS0 & $\mathrm{R} / \mathrm{NR}$ & $\mathrm{R} / \mathrm{NR}$ & $\mathrm{NR}$ & $\mathrm{R} / \mathrm{NR}$ & $\mathrm{NG}$ & $\mathrm{NG}$ \\
\hline RLA0 \& RLS0 & $\mathrm{NG}$ & $\mathrm{NG}$ & $\mathrm{NG}$ & $\mathrm{NG}$ & $\mathrm{NR}$ & $\mathrm{R} / \mathrm{NR}$ \\
\hline SHS0 \& RLA0 & $\mathrm{NG}$ & $\mathrm{NG}$ & $\mathrm{NG}$ & $\mathrm{NG}$ & $\mathrm{R} / \mathrm{NR}$ & $\mathrm{R} / \mathrm{NR}$
\end{tabular}

${ }^{a} \mathrm{R} / \mathrm{NR}$ : the interaction angles satisfying the resonance condition are numerically found for a certain range of primary frequencies. NR: no intersection angles satisfying the resonance condition are numerically found for any combinations of primary frequencies. $\mathrm{NG}$ : the secondary mode is not generated since $f_{m}^{\text {surf }}+f_{m}^{\text {vol }}=0$. 


\section{Figure captions}

Fig. 1 Homogeneous and isotropic elastic plate of thickness $2 h$. The plate has infinite length in the $X_{1}$ and $X_{2}$ directions.

Fig. 2 Dispersion curves of Rayleigh-Lamb and shear-horizontal waves for an aluminum alloy plate.

Fig. 3 Frequency dependence of intersection angles for the resonant interaction of the sum-frequency component. (a) SHSO and SHS0 interaction producing RLS0; (b) RLA0 and RLA0 interaction producing RLS0; (c) RLA0 and RLA0 interaction producing SHS0; (d) RLS0 and SHS0 interaction producing RLS0; (e) SHS0 and RLA0 interaction producing RLA0. The regions in white color have no intersection angles satisfying the resonance condition.

Fig. 4 Frequency dependence of intersection angles for the resonant interaction of the difference-frequency component. (a) RLS0 and RLS0 interaction producing SHS0; (b) RLA0 and RLA0 interaction producing SHS0; (c) RLS0 and SHS0 interaction producing RLS0; (d) RLS0 and SHS0 interaction producing SHS0; (e) RLA0 and RLS0 interaction producing RLA0; (f) SHS0 and RLA0 interaction producing RLA0. The regions in white color have no intersection angles satisfying the resonance condition or correspond to $\Omega^{(1)}-\Omega^{(2)}=0$, which is out of the frequency range in Eq. (28).

Fig. 5 Frequency dependence of intersection angles for the resonant interaction of bulk longitudinal (L) and shear (T) waves. (a) $\mathrm{T}$ and $\mathrm{T}$ interaction producing $\mathrm{L}$ with sum frequency; (b) L and $\mathrm{T}$ interaction producing $\mathrm{L}$ with sum frequency; (c) $\mathrm{L}$ and $\mathrm{L}$ interaction producing $\mathrm{T}$ with difference frequency; (d) $\mathrm{L}$ and $\mathrm{T}$ interaction producing $\mathrm{L}$ with difference frequency; (e) $\mathrm{L}$ and $\mathrm{T}$ interaction 
producing $\mathrm{T}$ with difference frequency. The regions in white color have no intersection angles satisfying the resonance condition or correspond to $\Omega^{(1)}-$ $\Omega^{(2)}=0$, which is out of the frequency range in Eq. (28).

Fig. 6 Effects of intersection angle on the RLS0 and SHS0 interaction. (a) Propagation angle of secondary wave; (b) wavenumber of secondary wave; (c) modal amplitude of the secondary RLS0 with sum frequency; (d) modal amplitude of the secondary RLS0 with difference frequency; (e) modal amplitude of the secondary SHSO with sum frequency; (f) modal amplitude of the secondary SHS0 with difference frequency. The primary frequencies are $\Omega^{(1)}=0.5$ and $\Omega^{(2)}$ $=0.2$ for the RLS0 and SHS0 modes, respectively.

Fig. 7 Effects of intersection angle on the SHS0 and RLA0 interaction. (a) Propagation angle of secondary wave; (b) wavenumber of secondary wave; (c) modal amplitude of the secondary RLA0 with sum frequency; (d) modal amplitude of the secondary RLA0 with difference frequency. The primary frequencies are $\Omega^{(1)}=0.2$ and $\Omega^{(2)}=0.7$ for the SHSO and RLA0 modes, respectively.

Fig. 8 Variation of the modal amplitudes of sum-frequency secondary wave with the intersection angle and the second primary frequency when $\Omega^{(1)}=0.5$ and $X_{1}{ }^{\prime} / h$ =100. (a) RLS0 produced by SHS0 and SHS0 interaction; (b) RLS0 produced by RLA0 and RLA0 interaction; (c) SHS0 produced by RLA0 and RLA0 interaction; (d) RLS0 produced by RLS0 and SHS0 interaction; (e) RLA0 produced by SHSO and RLA0 interaction. The dotted lines indicate the resonance conditions.

Fig. 9 Variation of the modal amplitudes of difference-frequency secondary wave with 
the intersection angle and the second primary frequency when $\Omega^{(1)}=0.5$ and $X_{1}{ }^{\prime} / h=100$. (a) SHS0 produced by RLS0 and RLS0 interaction; (b) SHS0 produced by RLA0 and RLA0 interaction; (c) RLS0 produced by RLS0 and SHS0 interaction; (d) SHS0 produced by RLS0 and SHS0 interaction; (e) RLA0 produced by RLA0 and RLS0 interaction; (f) RLA0 produced by SHS0 and RLA0 interaction. The dotted lines indicate the resonance conditions and the regions in white color correspond to $\Omega^{(1)}-\Omega^{(2)}=0$, for which the non-collinear interaction produces the static displacements. 

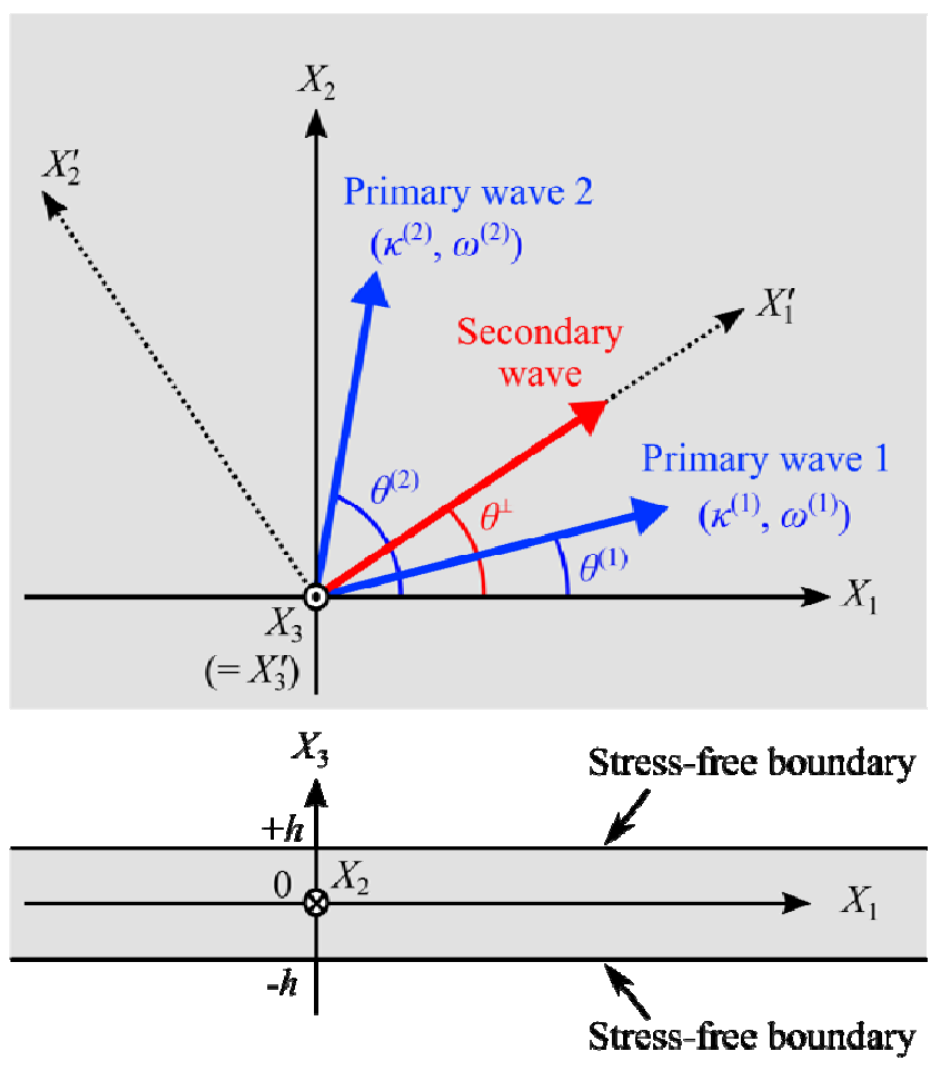

Fig. 1

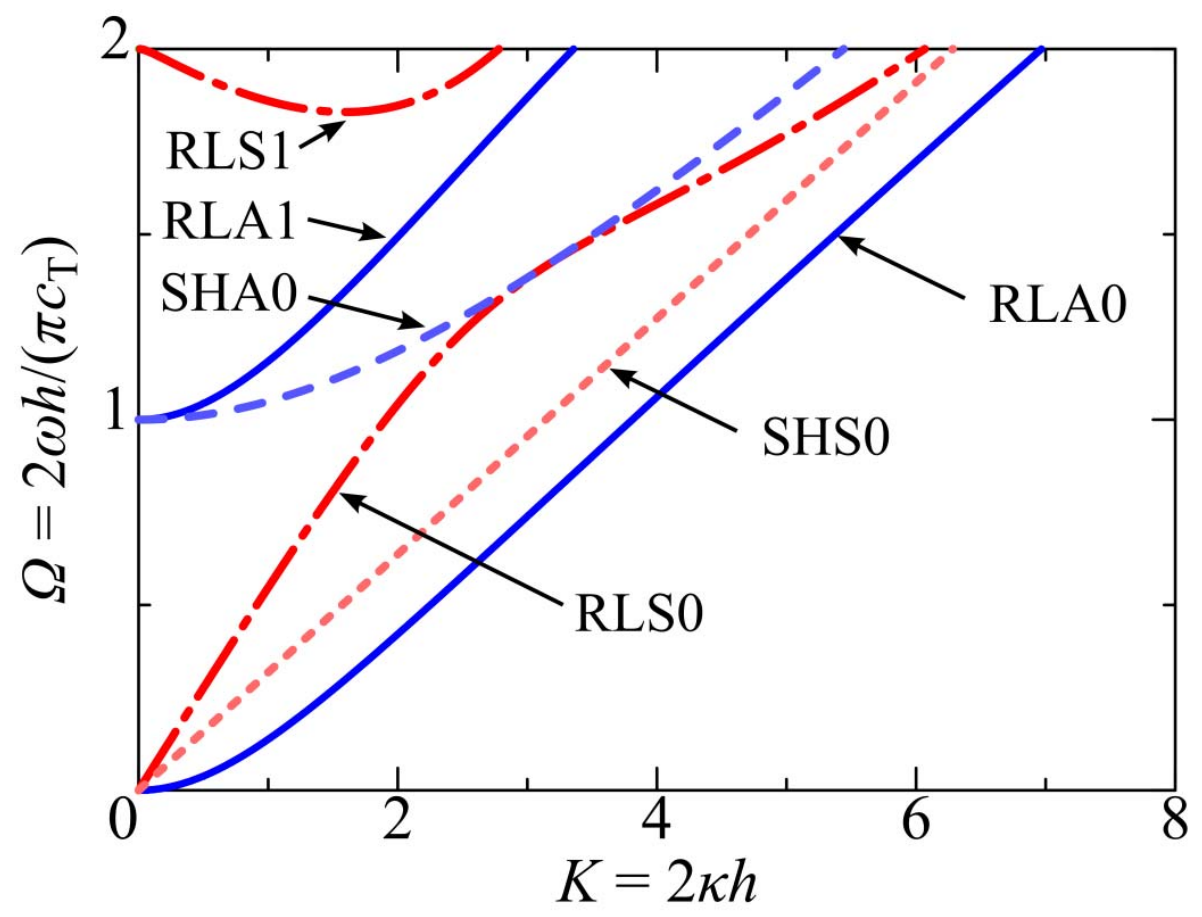

Fig. 2 


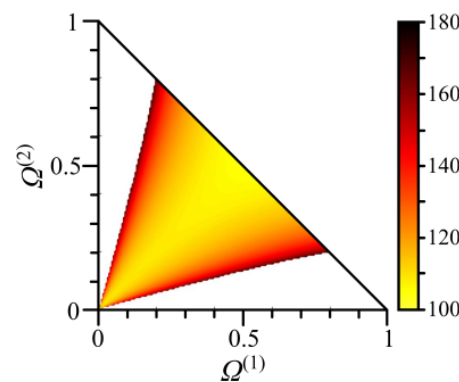

(a) $\operatorname{SHS} 0\left(\Omega^{(1)}\right) \& \operatorname{SHS} 0\left(\Omega^{(2)}\right)$ $\rightarrow \operatorname{RLS} 0\left(\Omega^{(1)}+\Omega^{(2)}\right)$

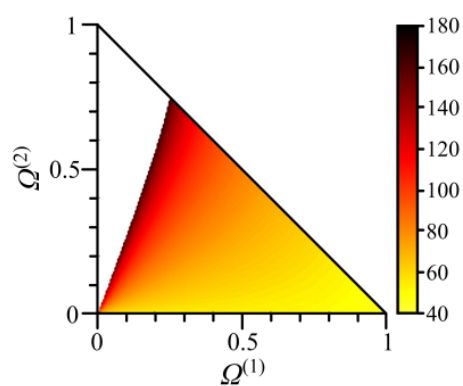

(d) RLS0 $\left(\Omega^{(1)}\right) \& \operatorname{SHSO}\left(\Omega^{(2)}\right)$ $\rightarrow \operatorname{RLS} 0\left(\Omega^{(1)}+\Omega^{(2)}\right)$

Fig. 3

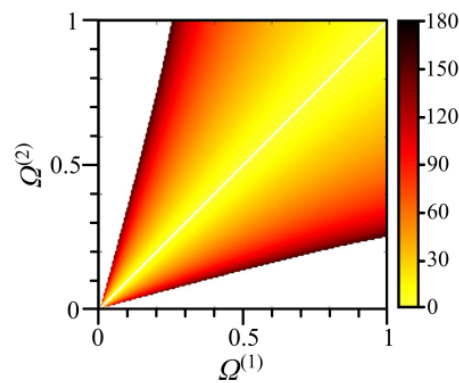

(a) $\operatorname{RLS} 0\left(\Omega^{(1)}\right) \& \operatorname{RLS} 0\left(\Omega^{(2)}\right)$ $\rightarrow \operatorname{SHS} 0\left(\left|\Omega^{(1)}-\Omega^{(2)}\right|\right)$

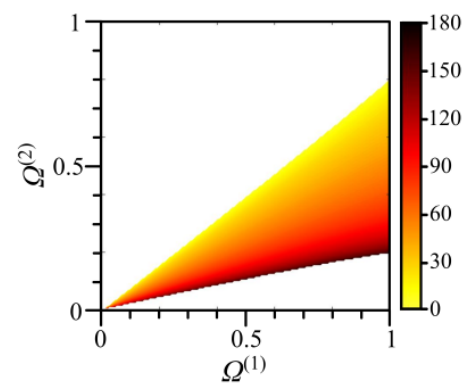

(d) $\operatorname{RLS} 0\left(\Omega^{(1)}\right) \& \operatorname{SHS} 0\left(\Omega^{(2)}\right)$ $\rightarrow \operatorname{SHS} 0\left(\left|\Omega^{(1)}-\Omega^{(2)}\right|\right)$

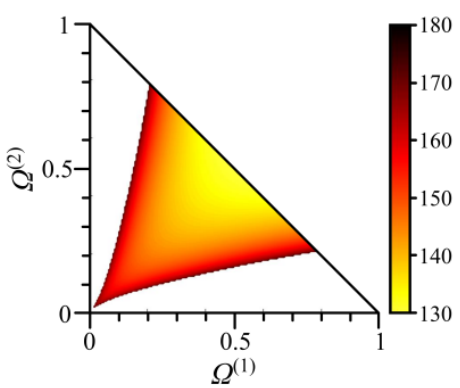

(b) $\operatorname{RLA0}\left(\Omega^{(1)}\right) \& \operatorname{RLA0}\left(\Omega^{(2)}\right)$ $\rightarrow \operatorname{RLS0}\left(\Omega^{(1)}+\Omega^{(2)}\right)$

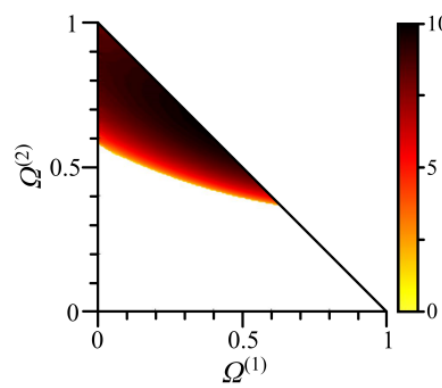

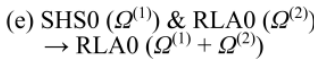

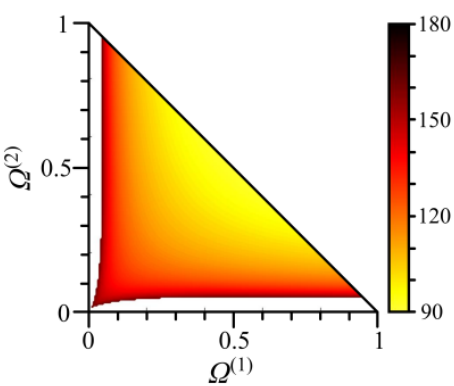

(c) RLA0 $\left(\Omega^{(1)}\right) \& \operatorname{RLA0}\left(\Omega^{(2)}\right)$ $\rightarrow \operatorname{SHS} 0\left(\Omega^{(1)}+\Omega^{(2)}\right)$

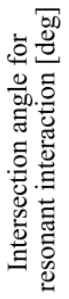

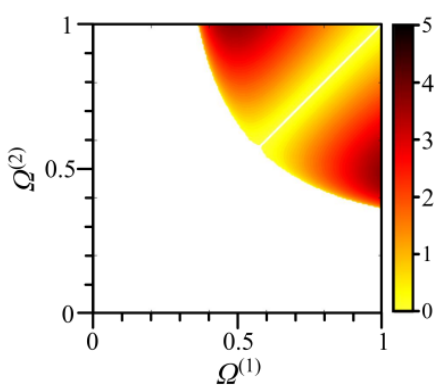

(b) RLA0 $\left(\Omega^{(1)}\right) \& \operatorname{RLA} 0\left(\Omega^{(2)}\right)$ $\rightarrow \operatorname{SHS0}\left(\left|\Omega^{(1)}-\Omega^{(2)}\right|\right)$

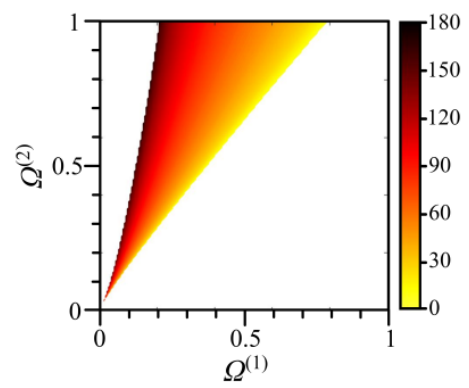

(e) $\operatorname{RLA} 0\left(\Omega^{(1)}\right) \& \operatorname{RLS} 0\left(\Omega^{(2)}\right)$ $\rightarrow \operatorname{RLA} 0\left(\left|\Omega^{(1)}-\Omega^{(2)}\right|\right)$

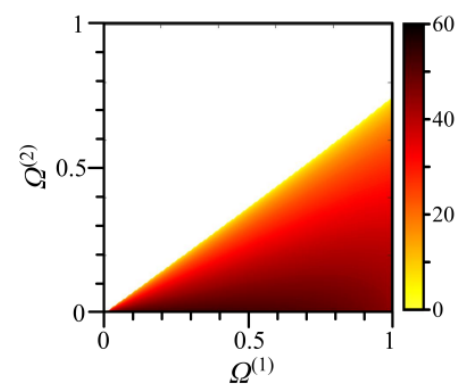

(c) $\operatorname{RLS} 0\left(\Omega^{(1)}\right) \& \operatorname{SHS} 0\left(\Omega^{(2)}\right.$ $\rightarrow \operatorname{RLS} 0\left(\left|\Omega^{(1)}-\Omega^{(2)}\right|\right)$

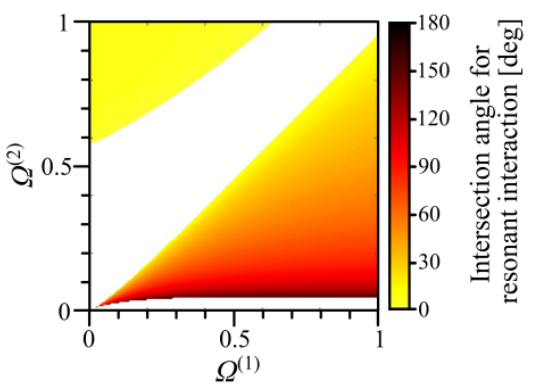

(f) $\operatorname{SHS} 0\left(\Omega^{(1)}\right) \& \operatorname{RLA0}\left(\Omega^{(2)}\right)$ $\rightarrow \operatorname{RLA} 0\left(\left|\Omega^{(1)}-\Omega^{(2)}\right|\right)$

Fig. 4 

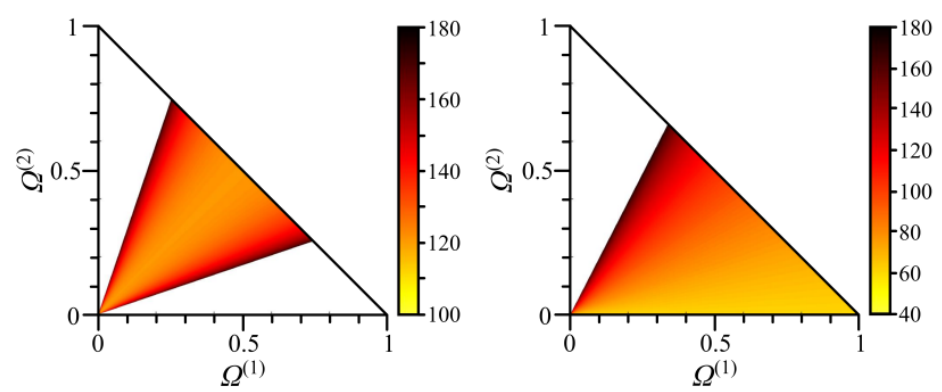

(a) $\mathrm{T}\left(\Omega^{(1)}\right) \& \mathrm{~T}\left(\Omega^{(2)}\right) \rightarrow \mathrm{L}\left(\Omega^{(1)}+\Omega^{(2)}\right)$

(b) $\mathrm{L}\left(\Omega^{(1)}\right) \& \mathrm{~T}\left(\Omega^{(2)}\right) \rightarrow \mathrm{L}\left(\Omega^{(1)}+\Omega^{(2)}\right)$

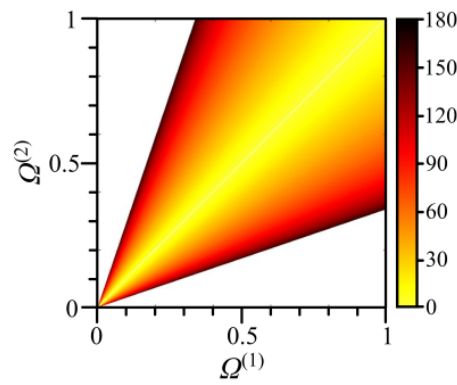

(c) $\mathrm{L}\left(\Omega^{(1)}\right) \& \mathrm{~L}\left(\Omega^{(1)}\right) \rightarrow \mathrm{T}\left(\left|\Omega^{(1)}-\Omega^{(2)}\right|\right)$

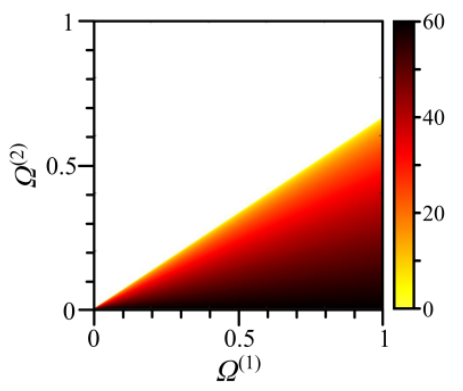

(d) $\mathrm{L}\left(\Omega^{(1)}\right) \& \mathrm{~T}\left(\Omega^{(2)}\right) \rightarrow \mathrm{L}\left(\left|\Omega^{(1)}-\Omega^{(2)}\right|\right)$

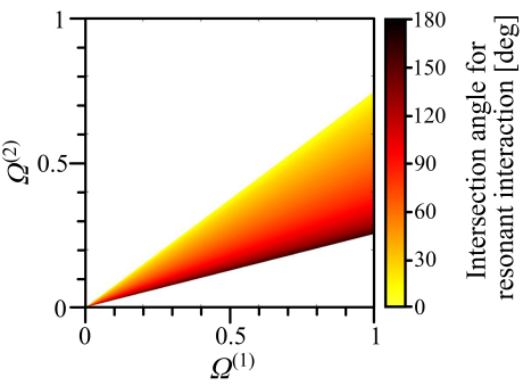

(e) $\mathrm{L}\left(\Omega^{(1)}\right) \& \mathrm{~T}\left(\Omega^{(2)}\right) \rightarrow \mathrm{T}\left(\left|\Omega^{(1)}-\Omega^{(2)}\right|\right)$

Fig. 5 

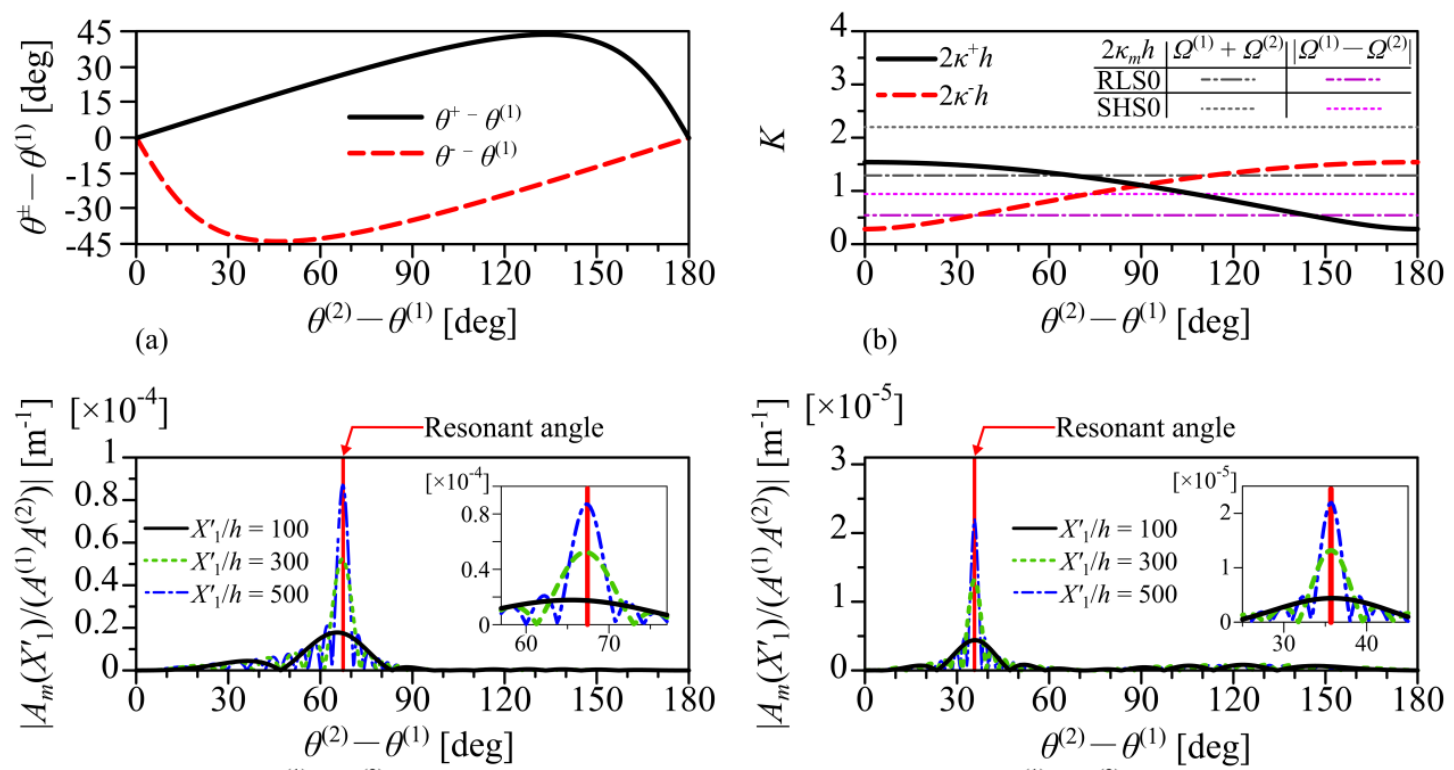

(c) $\operatorname{RLS} 0\left(\Omega^{(1)}+\Omega^{(2)}\right)$

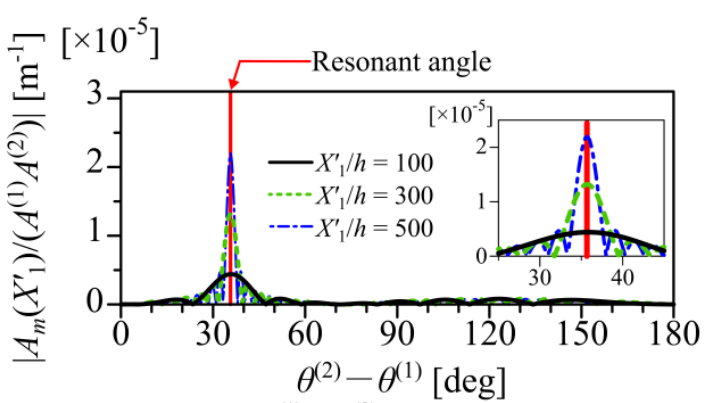

(d) RLS0 $\left(\left|\Omega^{(1)}-\Omega^{(2)}\right|\right)$

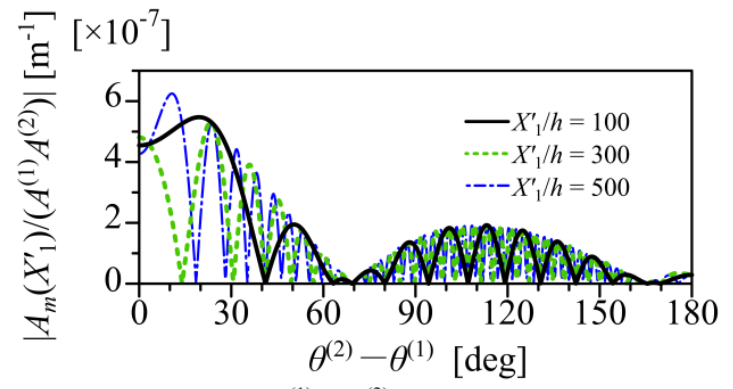

(e) $\operatorname{SHS} 0\left(\Omega^{(1)}+\Omega^{(2)}\right)$

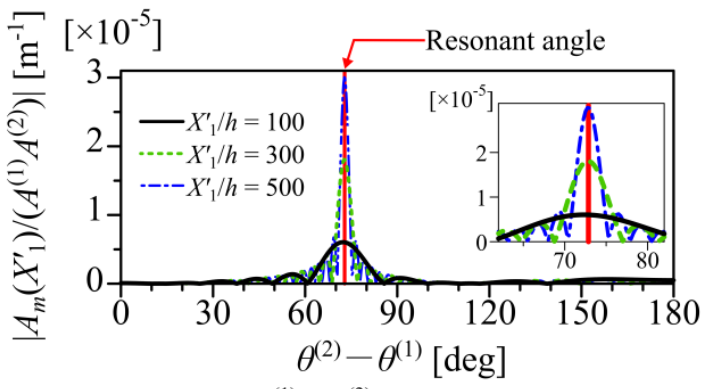

(f) SHS0 $\left(\left|\Omega^{(1)}-\Omega^{(2)}\right|\right)$

Fig. 6
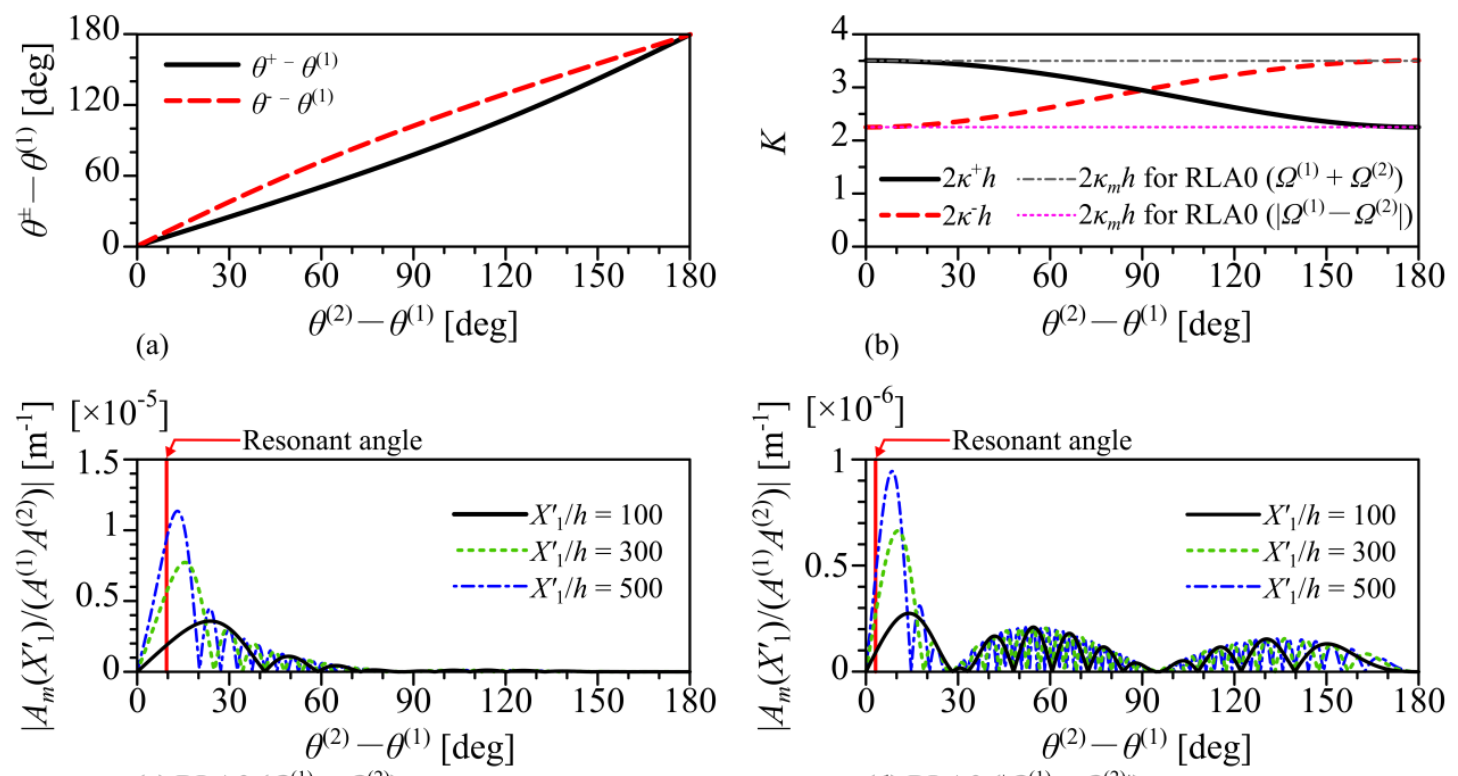

(c) RLA0 $\left(\Omega^{(1)}+\Omega^{(2)}\right)$

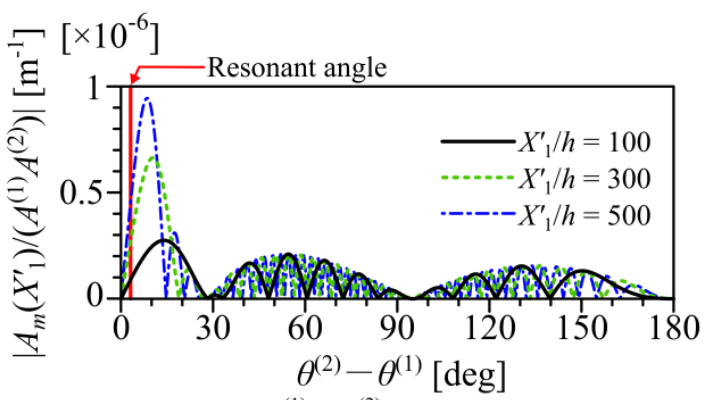

(d) $\operatorname{RLA0}\left(\left|\Omega^{(1)}-\Omega^{(2)}\right|\right)$

Fig. 7 


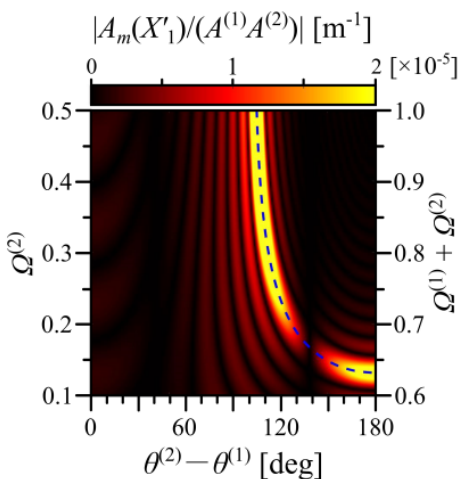

(a) $\underset{\operatorname{SHSO}}{\rightarrow}\left(\Omega^{(1)}\right) \& \operatorname{SHSO}\left(\Omega^{(2)}\right)$

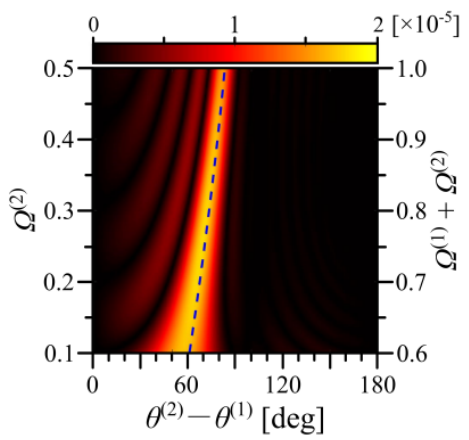

$\begin{aligned} \text { (d) } \operatorname{RLS0}\left(\Omega^{(1)}\right) \& \operatorname{SHS} 0\left(\Omega^{(2)}\right) & \rightarrow \operatorname{RLS0}\left(\Omega^{(1)}+\Omega^{(2)}\right) \\ & \rightarrow \log \end{aligned}$

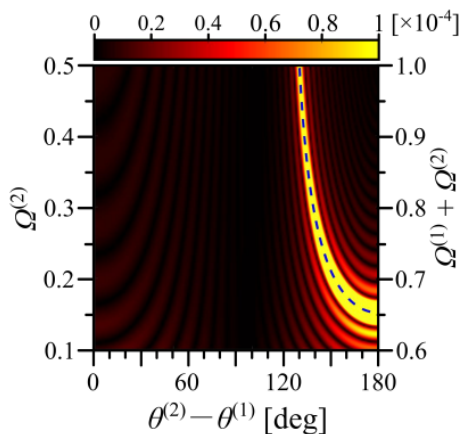

(b) $\operatorname{RLA} 0\left(\Omega^{(1)}\right) \& \operatorname{RLA} 0\left(\Omega^{(1)}\right)$ $\rightarrow \operatorname{RLSO}\left(\Omega^{(1)}+\Omega^{(2)}\right)$

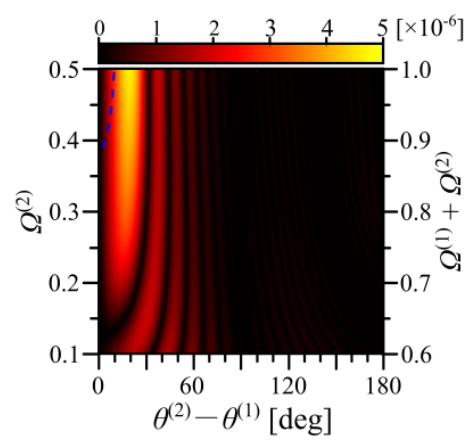

$\begin{aligned} \text { (e) } \operatorname{SHS} 0\left(\Omega^{(1)}\right) \& \operatorname{RLA0}\left(\Omega^{(2)}\right) & \rightarrow \operatorname{RLA}\left(\Omega^{(1)}+\Omega^{(1)}\right)\end{aligned}$

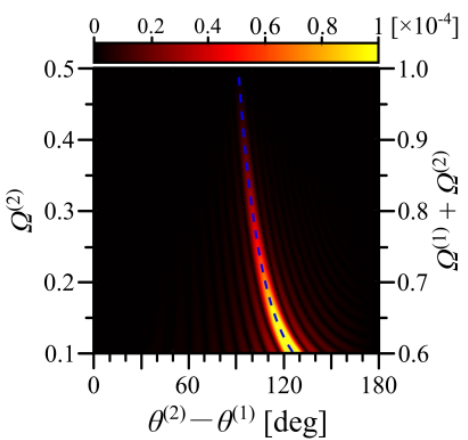

(c) $\operatorname{RLA} 0\left(\Omega^{(1)}\right) \& \operatorname{RLA} 0\left(\Omega^{(2)}\right)$ $\rightarrow \mathrm{SHSO}\left(\Omega^{(1)}+\Omega^{(2)}\right)$

Fig. 8 


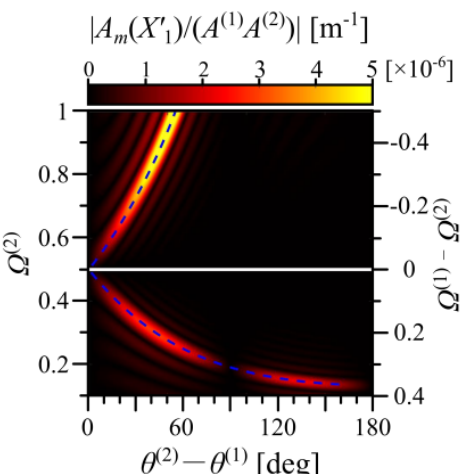

(a) $\operatorname{RLS} 0\left(\Omega^{(1)}\right) \& \operatorname{RLS} 0\left(\Omega^{(2)}\right)$ $\rightarrow$ SHS0 $\left(\left|\Omega^{(1)}-\Omega^{(2)}\right|\right)$

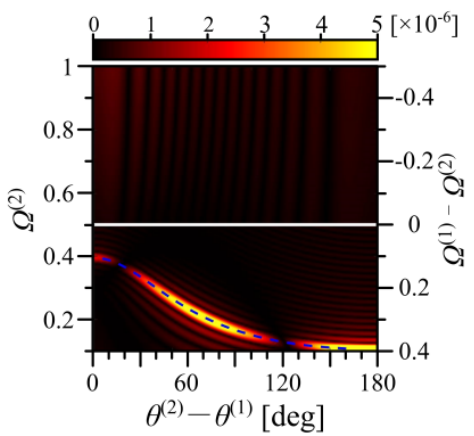

(d) $\operatorname{RLS} 0\left(\Omega^{(1)}\right) \& \operatorname{SHS} 0\left(\Omega^{(2)}\right.$ $\rightarrow \operatorname{SHS0}\left(\left|\Omega^{(1)}-\Omega^{(2)}\right|\right)$

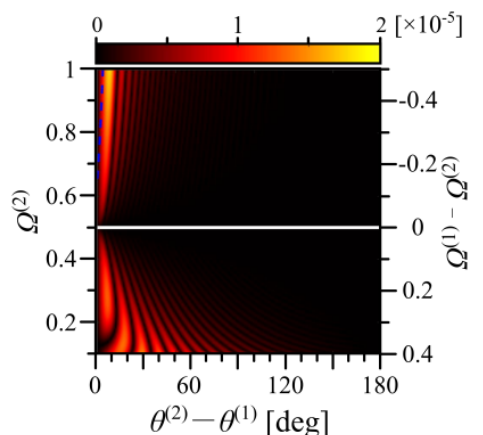

(b) $\operatorname{RLA0}\left(\Omega^{(1)}\right) \& \operatorname{RLA0}\left(\Omega^{(2)}\right)$ $\rightarrow \operatorname{SHS0}\left(\left|\Omega^{(1)}-\Omega^{(2)}\right|\right)$

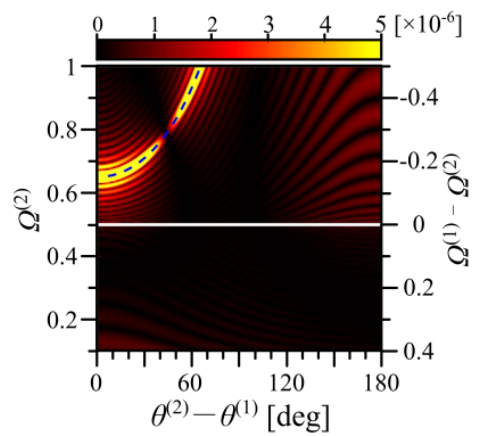

(e) $\operatorname{RLA0}\left(\Omega^{(1)}\right) \& \operatorname{RLS} 0\left(\Omega^{(2)}\right.$ $\rightarrow \operatorname{RLA} 0\left(\left|\Omega^{(1)}-\Omega^{(2)}\right|\right)$

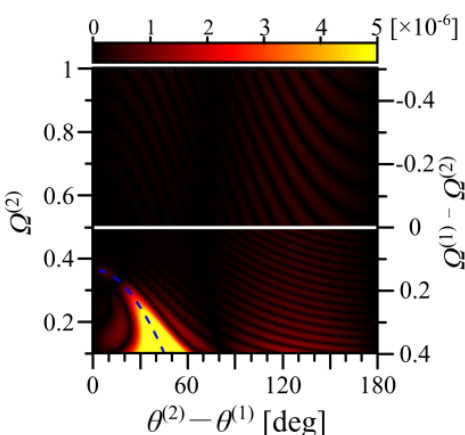

(c) $\operatorname{RLS} 0\left(\Omega^{(1)}\right) \& \operatorname{SHS} 0\left(\Omega^{(2)}\right)$
$\rightarrow \operatorname{RLS} 0\left(\left|\Omega^{(1)}-\Omega^{(2)}\right|\right)$

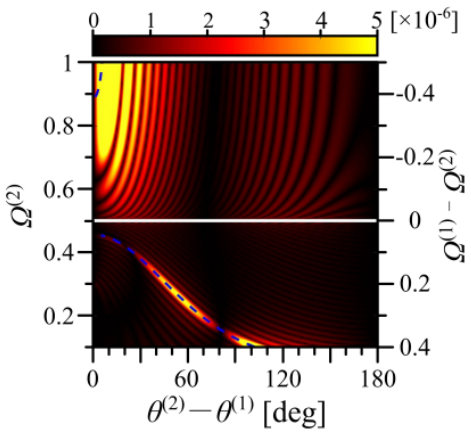

(f) SHS0 $\left(\Omega^{(1)}\right) \& \operatorname{RLA} 0\left(\Omega^{(2)}\right)$ $\rightarrow \operatorname{RLA0}\left(\left|\Omega^{(1)}-\Omega^{(2)}\right|\right)$

Fig. 9 